\title{
Extremal Values on the Sombor Index of Trees
}

\author{
Hanlin Chen ${ }^{1}$, Wenhao Li, Jing Wang \\ College of Computer Engineering and Applied Mathematics, \\ Changsha University, Changsha 410022, China
}

(Received April 9, 2021)

\begin{abstract}
The Sombor index of a graph $G$ is defined as

$$
S(G)=\sum_{u v \in E(G)} \sqrt{d_{G}(u)^{2}+d_{G}(v)^{2}}
$$

where $d_{G}(u)$ denote the degree of the vertex $u$ in $G$. In this article, we determine the extremal values of the Sombor index of trees with some given parameters, including matching number, pendant vertices, diameter, segment number, branching number, etc. The corresponding extremal trees are characterized completely.
\end{abstract}

\section{Introduction}

Let $G=(V(G), E(G))$ be a connected simple graph with order $|V(G)|=n$ and size $|E(G)|=m$. If a connected graph $T$ satisfies that $m=n-1$, then the graph $T$ is called a tree. Let $n_{i}(G)$ be the number of vertices of $G$ with degree $i$ and $m_{i, j}(G)$ the number of edges of $G$ joining a vertex of degree $i$ and a vertex of degree $j$. We will also use $n_{i}$ and $m_{i, j}$ for short when there is no risk of confusion. As usual, we denote the star and path on $n$ vertices by $S_{n}$ and $P_{n}$, respectively. Let $N_{G}(u)$, or $N(u)$ for short, be the neighbor set of the vertex $u \in V(G)$. For a vertex $u \in V(G)$, its degree $d_{G}(u)$ is equal to the number of vertices in $G$ adjacent to $u$, that is $d_{G}(u)=\left|N_{G}(u)\right|$. In particular, let $\Delta(G)=\max \left\{d_{G}(v): v \in V(G)\right\}$, i.e., the maximum degree of $G$. For $v \in V(G), G-v$ is the graph obtained from $G$ by deleting $v$ and its incident edges. If $X \subseteq E(G)$, then we use $G-X$ to denote the graph formed from $G$ by removing the edges in $X$. Similarly,

\footnotetext{
${ }^{1}$ Corresponding author: hlchen@ccsu.edu.cn (Hanlin Chen)
} 
$G+X$ denotes the graph obtained from $G$ by adding all edges in $X$. If $X=\{u v\}$, we will write $G-u v$ and $G+u v$ for simple. A vertex having degree one is said to be a pendant vertex and a vertex with degree greater than two is called a branching vertex. An edge having a pendant vertex as an endpoint is called a pendant edge. If $P=v_{0} v_{1} \ldots v_{k}$ is an induced sub-path of length $k$ of $G$ and $d_{G}\left(v_{0}\right)=1, d_{G}\left(v_{1}\right)=\cdots=d_{G}\left(v_{k-1}\right)=2$ and $d_{G}\left(v_{k}\right) \geq 3$, then we call $P$ a pendant path of $G$. We denote the set of all pendant vertices in graph $G$ by $P V(G)$. If a graph $G$ with vertex set $V(G)=\left\{d_{G}\left(v_{1}\right), d_{G}\left(v_{2}\right), \ldots, d_{G}\left(v_{n}\right)\right\}$ and $d_{G}\left(v_{1}\right) \geq d_{G}\left(v_{2}\right) \geq \cdots \geq d_{G}\left(v_{n}\right)$, then we call $\left(d_{G}\left(v_{1}\right), d_{G}\left(v_{2}\right), \ldots, d_{G}\left(v_{n}\right)\right)$ the degree sequence of $G$.

In order to predict some important physicochemical and bilogical properties of chemical compounds, hundreds of molecular structure descriptors (topological indices) have been introduced and studied by scholars from the field of mathematics or chemistry in the past decades. One of the most famous types is the so-called degree-based topological indices. The first Zagreb index, second Zagreb index, Randić index, Harmonic index, AtomBond-Connectivity index are all belong to this type. Nowadays, finding sharp bounds on degree-based topological indices for graphs under some given constrains has been one of the hottest research topics attracting many researches' attention. For some extremal results on degree-based topological indices, one may refer to $[1-4,6,8,12,13,15,16,18]$.

Motivated by the geometric interpretation of the degree radius, Gutman [11] recently put forward the Sombor index. The Sombor index of a graph $G$, denoted by $S(G)$, is defined as the sum of the weights of $\left(d_{G}(u)^{2}+d_{G}(v)^{2}\right)^{1 / 2}$ of all edges of $G$, that is

$$
S(G)=\sum_{u v \in E(G)} \sqrt{d_{G}(u)^{2}+d_{G}(v)^{2}} .
$$

It was shown that two degree-points have equal degree-radii if and only if they coincide, that is they share the same degree-coordinates, which is of great values and useful in chemical applications [11]. After reading the first paper on Sombor index, Deng, Tang and Wu in [10] found that the Sombor index is useful in predicting physico-chemical properties with high accuracy compared to some well-established and often used indices. They also obtain a sharp upper bound for the Sombor index among all molecular trees with fixed numbers of vertices. Very recently, Cruz, Gutman and Rada [9] obtained the extremal Sombor indices for chemical graphs, chemical trees and hexagonal systems. Extremal values on the Sombor index of unicyclic graphs and bicyclic graphs have been studied by Cruz and Gutman [7]. Wang, Mao, Li and Furtula [17] investigated the mathematical 
relations between the Sombor index and some other well-known degree-based descriptors. Moreover, some Nordhaus-Gaddum-type results are also obtained in their paper. Along this line, many extremal problems could be extended to the Sombor index.

The main goal of this paper is to derive some sharp bounds on the Sombor index of trees having some given parameters.

\section{Preliminaries}

In this section we give some useful lemmas which will be used frequently in the sequel section.

Lemma 2.1 Let $\phi(x, y)=\sqrt{x^{2}+y^{2}}-\sqrt{(x-1)^{2}+y^{2}}$, where $x>1$ and $y>0$, then the function $\phi(x, y)$ is strictly increasing with $x$ and strictly decreasing with $y$.

Proof. Note that $x^{2}\left[(x-1)^{2}+y^{2}\right]-(x-1)^{2}\left(x^{2}+y^{2}\right)=y^{2}(2 x-1)>0$ for $x>1$ and $y>0$, which means $x \sqrt{(x-1)^{2}+y^{2}}-(x-1) \sqrt{x^{2}+y^{2}}>0$ for $x>1$ and $y>0$, then we have

$$
\frac{\partial \phi(x, y)}{\partial x}=\frac{x}{\sqrt{x^{2}+y^{2}}}-\frac{x-1}{\sqrt{(x-1)^{2}+y^{2}}}=\frac{x \sqrt{(x-1)^{2}+y^{2}}-(x-1) \sqrt{x^{2}+y^{2}}}{\sqrt{\left(x^{2}+y^{2}\right)\left[(x-1)^{2}+y^{2}\right]}}>0
$$

and

$$
\frac{\partial \phi(x, y)}{\partial y}=\frac{y}{\sqrt{x^{2}+y^{2}}}-\frac{y}{\sqrt{(x-1)^{2}+y^{2}}}<0
$$

for $x>1$ and $y>0$. Thus we complete the proof.
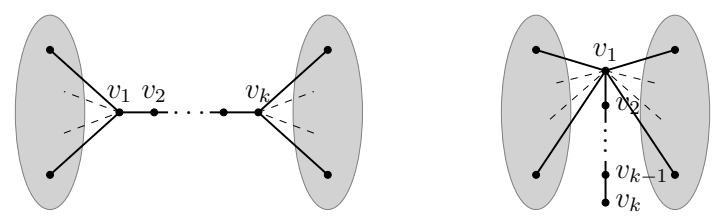

Figure 1. The path-lifting transformation: $G$ and $G^{\prime}$ in Lemma 2.2 .

Lemma 2.2 Let $G$ be a graph and $P=v_{1} v_{2} \ldots v_{k}$ an induced sub-path in $G$, where $d_{G}\left(v_{1}\right) \geq 2$ and $d_{G}\left(v_{k}\right) \geq 2 . G^{\prime}=G-\left\{v_{k} w: w \in N\left(v_{k}\right) \backslash\left\{v_{k-1}\right\}\right\}+\left\{v_{1} w: w \in\right.$ $\left.N\left(v_{k}\right) \backslash\left\{v_{k-1}\right\}\right\}$. The process from $G$ to $G^{\prime}$ will be called path-lifting transformation, see Figure 1. Then we have $S(G)<S\left(G^{\prime}\right)$. 
Proof. Let $\psi(x, y)=\sqrt{4+x^{2}}+\sqrt{4+y^{2}}-\sqrt{4+(x+y-1)^{2}}-\sqrt{5}$, where $x \geq 2$ and $y \geq 2$. Then it is easy to verify that

$$
\frac{\partial \psi(x, y)}{\partial x}=\frac{x}{\sqrt{4+x^{2}}}-\frac{x+y-1}{\sqrt{4+(x+y-1)^{2}}}=\frac{x \sqrt{4+(x+y-1)^{2}}-(x+y-1) \sqrt{4+x^{2}}}{\sqrt{\left(4+x^{2}\right)\left[\sqrt{4+(x+y-1)^{2}}\right]}}<0
$$

and

$$
\frac{\partial \psi(x, y)}{\partial y}=\frac{y}{\sqrt{4+y^{2}}}-\frac{x+y-1}{\sqrt{4+(x+y-1)^{2}}}=\frac{y \sqrt{4+(x+y-1)^{2}}-(x+y-1) \sqrt{4+y^{2}}}{\sqrt{\left(4+y^{2}\right)\left[\sqrt{4+(x+y-1)^{2}}\right]}}<0,
$$

implying the function $\psi(x, y)$ strictly decreases with $x \geq 2$ for fixed $y \geq 2$, and strictly decreases with $y \geq 2$ for fixed $x \geq 2$. For convenience, we let $d_{G}\left(v_{1}\right)=x \geq 2$ and $d_{G}\left(v_{k}\right)=y \geq 2$ in the following discussion.

Case 1. $k>2$.

Then it follows that

$$
\begin{aligned}
S(G)-S\left(G^{\prime}\right)= & \sqrt{2^{2}+x^{2}}+\sqrt{2^{2}+y^{2}}+\sum_{w \in N_{G}\left(v_{1}\right) \backslash\left\{v_{2}\right\}} \sqrt{x^{2}+d_{G}(w)^{2}} \\
& +\sum_{w \in N_{G}\left(v_{k}\right) \backslash\left\{v_{k-1}\right\}} \sqrt{y^{2}+d_{G}(w)^{2}}-\sqrt{1^{2}+2^{2}}-\sqrt{2^{2}+(x+y-1)^{2}} \\
& -\sum_{w \in N_{G}\left(v_{1}\right) \cup N_{G}\left(v_{k}\right) \backslash\left\{v_{2}, v_{k-1}\right\}} \sqrt{(x+y-1)^{2}+d_{G}(w)^{2}} \\
< & \sqrt{2^{2}+x^{2}}+\sqrt{2^{2}+y^{2}}-\sqrt{1^{2}+2^{2}}-\sqrt{2^{2}+(x+y-1)^{2}}=\psi(x, y) \\
\leq & \psi(2,2)=\sqrt{2^{2}+2^{2}}+\sqrt{2^{2}+2^{2}}-\sqrt{1^{2}+2^{2}}-\sqrt{2^{2}+(2+2-1)^{2}} \\
= & 4 \sqrt{2}-\sqrt{5}-\sqrt{13} \approx-0.1848<0,
\end{aligned}
$$

implying $S(G)<S\left(G^{\prime}\right)$.

Case 2. $k=2$.

Note first that $1+(x+y-1)^{2}-\left(x^{2}+y^{2}\right)=2(x y-x-y)+1>0$ for $x \geq 2$ and $y \geq 2$, then we have

$$
\begin{aligned}
S(G)-S\left(G^{\prime}\right)= & \sqrt{x^{2}+y^{2}}+\sum_{w \in N_{G}\left(v_{1}\right) \backslash\left\{v_{2}\right\}} \sqrt{x^{2}+d_{G}(w)^{2}}+\sum_{w \in N_{G}\left(v_{2}\right) \backslash\left\{v_{1}\right\}} \sqrt{y^{2}+d_{G}(w)^{2}} \\
& -\sqrt{1^{2}+(x+y-1)^{2}}-\sum_{w \in N_{G}\left(v_{1}\right) \cup N_{G}\left(v_{2}\right) \backslash\left\{v_{1}, v_{2}\right\}} \sqrt{(x+y-1)^{2}+d_{G}(w)^{2}} \\
< & \sqrt{x^{2}+y^{2}}-\sqrt{1+(x+y-1)^{2}}<0,
\end{aligned}
$$

which means $S(G)<S\left(G^{\prime}\right)$.

Thus, the proof is completed.

Applying the path-lifting transformation, the following result can be obtained immediately. 
Corollary 2.3 Let $T$ be a tree of order $n \geq 3$, then $2 \sqrt{2}(n-3)+2 \sqrt{5} \leq S(T) \leq$ $(n-1) \sqrt{n^{2}-2 n+2}$. The lower bound attains if and only if $T \cong P_{n}$ and the upper bound attains if and only if $T \cong S_{n}$.

Proof. Let $T$ be a tree different form the star $S_{n}$, then by finite steps of using the path-lifting transformation one can arrive the star $S_{n}$. Thus by Lemma 2.2 we have $S(T) \leq S\left(S_{n}\right)=(n-1) \sqrt{n^{2}-2 n+2}$. On the other hand, if $T \nRightarrow P_{n}$, then after proper times of applying the path-lifting transformation on $P_{n}$ one can get the desired tree $T$. Again by Lemma 2.2 we have $S(T) \geq S\left(P_{n}\right)=2 \sqrt{2}(n-3)+2 \sqrt{5}$.

\section{Main results}

In this section, we determine the extremal values of Sombor indices of trees with some given graphic parameters, such as matching number, pendant vertices, segments, branching vertices, and so forth.

\subsection{The maximum Sombor index of trees with a given matching number}

For an edge subset $M \subseteq E(G)$, if all edges in $M$ have no common endpoints, then we call $M$ is a matching of graph $G$. A matching $M$ is a maximum matching if there is no other matching $M^{\prime}$ of $G$ such that $\left|M^{\prime}\right|>|M|$. The number of edges in a maximum matching of graph $G$ is called the matching number of $G$, denoted by $\beta(G)$. A maximum matching $M$ with $|M|=\beta$ also said to be a $\beta$-matching. If $M$ is a matching, the two ends of each edge of $M$ are said to be matched under $M$, and each vertex incident with an edge of $M$ is said to be an $M$-saturated. If every vertex of $G$ is $M$-saturated, then we call $M$ a perfect matching. Let $\mathscr{T}_{n, \beta}$ be the set of trees with $n$ vertices and matching number $\beta$. Let $T_{n}^{\beta}$ be the tree obtained from the star $S_{n-\beta+1}$ by subdividing $\beta-1$ pendant edges in $S_{n-\beta+1}$. It is obvious that $T_{n}^{\beta} \in \mathscr{T}_{n, \beta}$ and it has a perfect matching for $n=2 \beta$. The two trees $T_{n}^{\beta}$ and $T_{2 \beta}^{\beta}$ are illustrated in Figure 2 .

Lemma $3.1[5,14]$ (i) If $T \in \mathscr{T}_{2 \beta, \beta}$ and $\beta \geq 2$, then $T$ has a pendant vertex whose unique neighbour is of degree two. (ii) If $T \in \mathscr{T}_{n, \beta}$ and $n>2 \beta$, then there is a $\beta$-matching $M$ and a pendant vertex $v$ such that $M$ does not saturate $v$. 


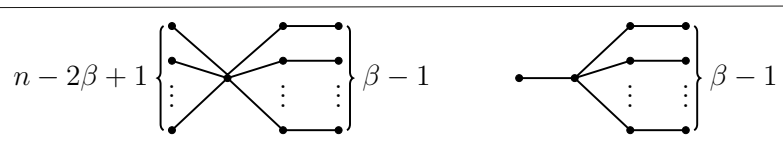

Figure 2. The trees $T_{n}^{\beta}$ (left) and $T_{2 \beta}^{\beta}$ (right).

Lemma 3.2 Let $T \in \mathscr{T}_{n, \beta}$ with the maximum Sombor index and $M$ is a $\beta$-matching of $T$.

(i) If $e=u v \in M$, then e is a pendant edge of $T$.

(ii) If $v$ is not a pendant vertex of $T$, then $v$ is $M$-saturated.

Proof. (i) Suppose to the contrary that $e=u v \in M$ but it is not a pendant edge of $T$. Let $T^{\prime}=T-\left\{u w: w \in N_{T}(u) \backslash\{v\}\right\}+\left\{v w: w \in N_{T}(u) \backslash\{v\}\right\}$. It is clear that $T^{\prime} \in \mathscr{T}_{n, \beta}$. By Lemma 2.2 we have $S\left(T^{\prime}\right)>S(T)$, a contradiction to the choice of $T$. (ii) If $T \cong S_{n}$, then the result follows immediately. If $T \neq S_{n}$ and $v$ is not a pendant vertex in $T$, then we can see that there is a vertex $u \in N_{T}(v)$ and $u$ is not a pendant vertex. If $v$ is not $M$-saturated, then we have $u v \notin M$. Let $T^{\prime \prime}=T-\left\{u w: w \in N_{T}(u) \backslash\{v\}\right\}+\left\{v w: w \in N_{T}(u) \backslash\{v\}\right\}$, and it is easy to see that $T^{\prime \prime} \in \mathscr{T}_{n, \beta}$. By Lemma 2.2 we have $S\left(T^{\prime}\right)>S(T)$, a contradiction to the choice of $T$.

Theorem 3.3 Let $T \in \mathscr{T}_{2 \beta, \beta}$, then $S(T) \leq \sqrt{1+\beta^{2}}+(\beta-1)\left(\sqrt{5}+\sqrt{4+\beta^{2}}\right)$ with equality if and only if $T \cong T_{2 \beta}^{\beta}$.

Proof. We prove the result by applying induction on $\beta$. If $\beta=1,2$, then we have $T \cong T_{2}^{1} \cong P_{2}, T \cong T_{4}^{2} \cong P_{4}$. Therefore, the result is true for $\beta=1$ and $\beta=2$. Suppose that it is true for $T \in \mathscr{T}_{2 k, k}, k=1,2, \ldots, \beta-1$. We set $T \in \mathscr{T}_{2 \beta, \beta}$ such that $S(T)$ is maximum. Let $M$ be a perfect matching of $T$. By Lemma 3.1 (i) there is a pendant vertex $u$ in $T$ adjacent to a vertex $v$ of degree two. Then, $u v \in M$. Let $T^{\prime}=T-u-v$, then $T^{\prime} \in \mathscr{T}_{2 \beta-2, \beta-1}$. By induction hypothesis, we have $S\left(T^{\prime}\right) \leq S\left(T_{2 \beta-2}^{\beta-1}\right)$. Let $w \in N(v) \backslash\{u\}$. Assume that $d_{T}(w)=x, N_{T}(w)=\left\{w_{1}, w_{2}, \ldots, w_{x-1}, v\right\}$ and $d_{T}\left(w_{i}\right)=t_{i}$, then $d_{T^{\prime}}(w)=$ $x-1$ and $d_{T^{\prime}}\left(w_{i}\right)=t_{i}$ for $i=1,2, \ldots, x-1$. By Lemma 3.2 (i), there exist at least $\beta$ pendant vertices in $T$. Note also that $T$ has a perfect matching. Hence there is at most one neighbors of $w$ with degree one. Thus, there exist at least $\beta-1$ pendant vertices not adjacent to $w$ in $T$, that is, $x \leq 2 \beta-1-(\beta-1)=\beta$. Moveover, since $w$ is not a 
pendant vertex of $T$, by Lemma 3.2, there exists some $t_{i}=1$. Without loss of generality, we assume that $t_{1}=1$ and $t_{2}, t_{3}, \ldots, t_{x-1} \geq 2$.

$$
\begin{aligned}
S(T)= & S\left(T^{\prime}\right)+\sqrt{1^{2}+2^{2}}+\sqrt{2^{2}+x^{2}}+\sum_{i=1}^{x-1} \sqrt{t_{i}^{2}+x^{2}}-\sum_{i=1}^{l-1} \sqrt{t_{i}^{2}+(x-1)^{2}} \\
= & S\left(T^{\prime}\right)+\sqrt{5}+\sqrt{4+x^{2}}+\sum_{i=1}^{x-1}\left[\sqrt{t_{i}^{2}+x^{2}}-\sqrt{t_{i}^{2}+(x-1)^{2}}\right] \\
\leq & S\left(T_{2 \beta-2}^{\beta-1}\right)+\sqrt{5}+\sqrt{4+x^{2}}+\sqrt{1+x^{2}}-\sqrt{1+(x-1)^{2}} \\
& +(x-2)\left[\sqrt{4+x^{2}}-\sqrt{4+(x-1)^{2}}\right] \\
= & S\left(T_{2 \beta-2}^{\beta-1}\right)+\sqrt{5}+\sqrt{4+x^{2}}+\phi(x, 1)+(x-2) \phi(x, 2) \\
\leq & S\left(T_{2 \beta-2}^{\beta-1}\right)+\sqrt{5}+\sqrt{4+\beta^{2}}+\phi(\beta, 1)+(\beta-2) \phi(\beta, 2) \\
= & \sqrt{1+\beta^{2}}+(\beta-1)\left(\sqrt{5}+\sqrt{4+\beta^{2}}\right) .
\end{aligned}
$$

The equalities above hold simultaneously if and only if $T^{\prime} \cong T_{2 \beta-2}^{\beta-1}, t_{1}=1$ and $t_{2}=t_{3}=$ $\cdots=t_{x-1}=2$ and $x=\beta$, which means that $T \cong T_{2 \beta}^{\beta}$. Thus, the proof is completed.

Theorem 3.4 Let $T \in \mathscr{T}_{n, \beta}$, then $S(T) \leq(n-2 \beta+1) \sqrt{1+(n-\beta)^{2}}+(\beta-1)[\sqrt{5}+$ $\left.\sqrt{4+(n-\beta)^{2}}\right]$ with equality if and only if $T \cong T_{n}^{\beta}$.

Proof. If $n=2,3$, then $T \cong T_{2}^{1}=P_{2}$ and $T \cong T_{3}^{1}=P_{3}$, respectively. Thus the result is true for $n=1,2$. If $n=2 \beta$, then the result holds by Theorem 3.3. Next, we suppose the result holds for all trees in $\mathscr{T}_{t, \beta}$ with $t \leq n-1$ and $t>2 \beta$. Let $T$ be a tree in $\mathscr{T}_{n, \beta}$ with maximum Sombor index and $M$ a $\beta$-matching of $T$. By Lemma 3.1 (ii) there exists a pendant vertex $v$ such that $T-v$ also contains a $\beta$-matching. Let $w$ be the unique neighbor of $v, d_{T}(w)=x$ and $N_{T}(w) \backslash\{v\}=\left\{w_{1}, w_{2}, \ldots, w_{x-1}\right\}$. Set $T^{\prime}=T-v$, then it is obvious that $T^{\prime} \in \mathscr{T}_{n-1, \beta}$. Moreover, we have $x \leq n-1-(\beta-1)=n-\beta$ since there are at least $\beta-1$ vertices saturated by $M$ in $V(T) \backslash\left\{w, v, w_{1}, w_{2}, \ldots, w_{x-1}\right\}$. Without loss of generality, we assume that $d_{T}\left(w_{1}\right)=d_{T}\left(w_{2}\right)=\cdots=d_{T}\left(w_{s}\right)=1$ and $d_{T}\left(w_{k}\right) \geq 2$ for $s+1 \leq k \leq x-1$. By Lemma 3.2 we know that every matching edge of $T$ is a pendant edge and every non-pendant vertex is $M$-saturated. Hence, the number of pendant vertices of $T$ is $n-\beta$. Thus, $s+1 \leq n-\beta-(\beta-1)=n-2 \beta$, i.e., $s \leq n-2 \beta$. By Lemma 2.1 and induction hypothesis, we have

$$
S(T)=S\left(T^{\prime}\right)+\sqrt{1^{2}+x^{2}}+\sum_{i=1}^{x-1}\left[\sqrt{d_{T}\left(w_{i}\right)^{2}+x^{2}}-\sqrt{d_{T}\left(w_{i}\right)^{2}+(x-1)^{2}}\right]
$$




$$
\begin{aligned}
= & S\left(T^{\prime}\right)+\sqrt{1+x^{2}}+s\left[\sqrt{1+x^{2}}-\sqrt{1+(x-1)^{2}}\right]+\sum_{i=s+1}^{x-1} \phi\left(x, d_{T}\left(w_{i}\right)\right) \\
\leq & S\left(T^{\prime}\right)+\sqrt{1+x^{2}}+s \cdot \phi(x, 1)+\sum_{i=s+1}^{x-1} \phi(x, 2) \\
= & S\left(T^{\prime}\right)+\sqrt{1+x^{2}}+s \cdot \phi(x, 1)+(x-s-1) \phi(x, 2) \\
\leq & S\left(T^{\prime}\right)+\sqrt{1+(n-\beta)^{2}}+s \cdot \phi(n-\beta, 1)+(n-\beta-s-1) \phi(n-\beta, 2) \\
\leq & S\left(T_{n-1}^{\beta}\right)+\sqrt{1+(n-\beta)^{2}}+s \cdot \phi(n-\beta, 1)+(n-\beta-s-1) \phi(n-\beta, 2) \\
= & S\left(T_{n-1}^{\beta}\right)+\sqrt{1+(n-\beta)^{2}}+(n-\beta-1) \phi(n-\beta, 2)+s[\phi(n-\beta, 1)-\phi(n-\beta, 2)] \\
\leq & S\left(T_{n-1}^{\beta}\right)+\sqrt{1+(n-\beta)^{2}}+(n-\beta-1) \phi(n-\beta, 2) \\
& +(n-2 \beta)[\phi(n-\beta, 1)-\phi(n-\beta, 2)] \\
= & (n-2 \beta+1) \sqrt{1+(n-\beta)^{2}}+(\beta-1)\left[\sqrt{5}+\sqrt{4+(n-\beta)^{2}}\right] .
\end{aligned}
$$

The equalities above hold simultaneously if and only if $T^{\prime} \cong T_{n-1}^{\beta}, x=n-\beta, s=n-2 \beta$ and $d_{T}\left(w_{k}\right)=2$ for $n-2 \beta+1 \leq k \leq n-\beta-1$, which means $T \cong T_{n}^{\beta}$.

\subsection{The maximum Sombor index of trees with given number of pendant vertices}

Let $\mathscr{H}_{n, p}$ be the set of trees with $n$ vertices in which there are $p$ pendant vertices. Let $Y_{n, p}$ be the broom graph, i.e., the tree obtained from the star $S_{p}$ by replacing one of its pendant edges by a path of length $n-p$. The following result shows that the boom $Y_{n, p}$ is the unique tree in $\mathscr{H}_{n, p}$ with the maximum Sombor index.

Theorem 3.5 Let $T$ be a tree in $\mathscr{H}_{n, p}$, then $S(T) \leq(p-1) \sqrt{1+p^{2}}+\sqrt{4+p^{2}}+2 \sqrt{2}(n-$ $p-1)+\sqrt{5}$ with equality if and only if $T \cong Y_{n, p}$.

Proof. If $p=2$ or $p=n-1$, then $T \cong P_{n}=Y_{n, 2}$ or $T \cong S_{n}=Y_{n, n-1}$. Thus, the result is true for $p=2$ and $n-1$. Now, we assume that $3 \leq p \leq n-2$ and the result holds for the trees in $\mathscr{H}_{n^{\prime}, p^{\prime}}$, where $n^{\prime} \leq n-1$ and $p^{\prime} \leq p-1$. Let $T \in \mathscr{H}_{n, p}$ and $v \in P V(T)$. If $u$ is the neighbor of $v$ and $N_{T}(u)=\left\{v, u_{1}, u_{2}, \ldots, u_{t-1}\right\}$, then $d_{T}(u)=t \leq p$ and there is at least one vertex in $N_{T}(u)$ with degree greater than two. Without loss of generality, we assume that $d_{T}\left(u_{1}\right) \geq 2$ and $d_{T}\left(u_{i}\right) \geq 1, i=1,2, \ldots, t-1$. Now, we set $T^{\prime}=T-v$.

If $d_{T}(u)=2$, then $d_{T^{\prime}}(u)=1$ and $T^{\prime} \in \mathscr{H}_{n-1, p}$. By induction hypothesis, we get $S\left(T^{\prime}\right) \leq S\left(Y_{n-1, p}\right)=(p-1) \sqrt{1+p^{2}}+\sqrt{4+p^{2}}+2 \sqrt{2}(n-p-2)+\sqrt{5}$. Thus, we have

$$
S(T)=S\left(T^{\prime}\right)+\sqrt{5}+\sqrt{d_{T}\left(u_{1}\right)^{2}+2^{2}}-\sqrt{d_{T}\left(u_{1}\right)^{2}+1^{2}}
$$




$$
\begin{aligned}
& \leq(p-1) \sqrt{1+p^{2}}+\sqrt{4+p^{2}}+2 \sqrt{2}(n-p-2)+\sqrt{5}+2 \sqrt{2}-\sqrt{5} \\
& =(p-1) \sqrt{1+p^{2}}+\sqrt{4+p^{2}}+2 \sqrt{2}(n-p-1)+\sqrt{5} .
\end{aligned}
$$

The equality holds if and only if $T^{\prime}=Y_{n, p-1}$ and $d_{T}\left(u_{1}\right)=2$, which implies that $T \cong Y_{n, p}$.

If $d_{T}(u) \geq 3$, then $d_{T^{\prime}}(u) \geq 2$ and $T^{\prime} \in \mathscr{H}_{n-1, p-1}$. By induction hypothesis, we have

$$
\begin{aligned}
S(T)= & S\left(T^{\prime}\right)+\sqrt{t^{2}+1^{2}}+\sum_{i=1}^{l-1} \sqrt{t^{2}+d_{T}\left(u_{i}\right)^{2}}-\sum_{i=1}^{t-1} \sqrt{(t-1)^{2}+d_{T}\left(u_{i}\right)^{2}} \\
\leq & S\left(Y_{n-1, p-1}\right)+\sqrt{t^{2}+1^{2}}+\sum_{i=1}^{t-1}\left[\sqrt{t^{2}+d_{T}\left(u_{i}\right)^{2}}-\sqrt{(t-1)^{2}+d_{T}\left(u_{i}\right)^{2}}\right] \\
\leq & S\left(Y_{n-1, p-1}\right)+\sqrt{t^{2}+1^{2}}+(t-2)\left(\sqrt{t^{2}+1}-\sqrt{(t-1)^{2}+1}\right) \\
& +\sqrt{t^{2}+4}-\sqrt{(t-1)^{2}+4} \\
= & S\left(Y_{n-1, p-1}\right)+\sqrt{t^{2}+1}+(t-2) \phi(t, 1)+\phi(t, 2) \\
\leq & S\left(Y_{n-1, p-1}\right)+\sqrt{p^{2}+1}+(p-2) \phi(p, 1)+\phi(p, 2) \quad \text { since } t \leq p \\
= & (p-1) \sqrt{1+p^{2}}+\sqrt{4+p^{2}}+2 \sqrt{2}(n-p-1)+\sqrt{5} .
\end{aligned}
$$

The equalities above hold simultaneously if and only if $T^{\prime} \cong Y_{n-1, p-1}, d_{T}\left(u_{1}\right)=d_{T}\left(u_{2}\right)=$ $\cdots=d_{T}\left(u_{t-1}\right)=1$ and $t=p$. Thus, we get $T \cong Y_{n, p}$, completing the proof.

\subsection{The maximum Sombor index of trees with a given biparti- tion}

If $G$ is a bipartite graph with $n$ vertices and its vertices can be partitioned into two parts $V_{p}$ and $V_{q}$ such that $\left|V_{p}\right|=p,\left|V_{q}\right|=q$ and there are no edges joining pairs of vertices belong to the same part of its partition, then we say that $G$ possesses a $(p, q)$-partition. Let $\mathscr{B}_{p, q}$ be the set of trees with $n$ vertices and a $(p, q)$-partition.

Let $H_{\ell}(s, t)$ be the tree of order $n$ obtained from a path $P_{\ell}=v_{1} v_{2} \ldots v_{\ell}$ by attaching $s$ and $t$ pendant vertices to each terminal vertices of $P_{\ell}$ respectively, where $s+t=n-\ell$, $s \geq t \geq 1$ and $\ell \geq 2$. $H_{\ell}(s, t)$ is, sometimes, also called double broom graph. If $\ell=2$, then we call $H_{2}(s, t)$ as a double star, and denote it by $S_{s, t}$.

Lemma 3.6 $S\left(H_{\ell}(n-\ell-1,1)\right)>S\left(H_{\ell}(n-\ell-2,2)\right)>\cdots>S\left(H_{\ell}\left(\left\lceil\frac{n-\ell}{2}\right\rceil,\left\lfloor\frac{n-\ell}{2}\right\rfloor\right)\right)$.

Proof. Let $T=H_{\ell}(s, t)$. it is suffices to show that $S\left(H_{\ell}(s, t)\right)>S\left(H_{\ell}(s-1, t+1)\right)$ for $s \geq t+2$. For convenience, let $T^{\prime}=H_{\ell}(s-1, t+1)$. Then, $d_{T}\left(v_{1}\right)=s+1, d_{T}\left(v_{\ell}\right)=t+1$, $d_{T^{\prime}}\left(v_{1}\right)=s$ and $d_{T^{\prime}}\left(v_{\ell}\right)=t+2$. 
If $\ell=2$, then by the definition of Sombor index we have

$$
\begin{aligned}
S(T)-S\left(T^{\prime}\right)= & s \sqrt{1^{2}+(s+1)^{2}}+\sqrt{(s+1)^{2}+(t+1)^{2}}+t \sqrt{1^{2}+(t+1)^{2}} \\
& -(s-1) \sqrt{1^{2}+s^{2}}-\sqrt{s^{2}+(t+2)^{2}}-(t+1) \sqrt{1^{2}+(t+2)^{2}} \\
= & (s-1) \phi(s+1,1)+\sqrt{(s+1)^{2}+(t+1)^{2}}-\sqrt{s^{2}+(t+2)^{2}} \\
& -t \cdot \phi(t+2,1)+\sqrt{1^{2}+(s+1)^{2}}-\sqrt{1^{2}+(t+2)^{2}} \\
> & (s-1) \phi(s+1,1)-t \cdot \phi(t+2,1) \\
> & {[\phi(s+1,1)-\phi(t+2,1)] t>0 . }
\end{aligned}
$$

If $\ell \geq 3$, then

$$
\begin{aligned}
S(T)-S\left(T^{\prime}\right)= & s \sqrt{1^{2}+(s+1)^{2}}+\sqrt{(s+1)^{2}+2^{2}}+\sqrt{(t+1)^{2}+2^{2}}+t \sqrt{1^{2}+(t+1)^{2}} \\
& -(s-1) \sqrt{1^{2}+s^{2}}-\sqrt{s^{2}+2^{2}}-\sqrt{2^{2}+(t+2)^{2}}-(t+1) \sqrt{1^{2}+(t+2)^{2}} \\
= & (s-1) \phi(s+1,1)+\sqrt{(s+1)^{2}+2^{2}}+\sqrt{(t+1)^{2}+2^{2}}-\sqrt{s^{2}+2^{2}} \\
& -\sqrt{2^{2}+(t+2)^{2}}-t \cdot \phi(t+2,1)+\sqrt{1^{2}+(s+1)^{2}}-\sqrt{1^{2}+(t+2)^{2}} \\
> & (s-1) \phi(s+1,1)-t \cdot \phi(t+2,1)+\phi(s+1,2)-\phi(t+2,2) \\
> & {[\phi(s+1,1)-\phi(t+2,1)] t-\phi(t+2,2)+\phi(s+1,2)>0 . }
\end{aligned}
$$

Thus, we get the desired result, completing the proof.

Theorem 3.7 Let $T$ be a tree in $\mathscr{B}_{p, q}$, then $S(T) \leq \sqrt{p^{2}+q^{2}}+(p-1) \sqrt{1+p^{2}}+(q-$ 1) $\sqrt{1+q^{2}}$, with equality if and only if $T \cong S_{p, q}$.

Proof. If $p=1$, then the unique tree in $\mathscr{B}_{p, q}$ is $S_{1, n-1}$. If $p=2$, then $\mathscr{B}_{2, n-2}=\left\{S_{2, n-2}\right\} \cup$ $\left\{H_{3}(s, t): s+t=n-3\right.$ and $\left.s \geq t \geq 1\right\}$. By and Lemmas 2.2 and 3.6 we have

$$
S\left(T_{2, n-2}\right)>S\left(H_{3}(n-4,1)\right)>S\left(H_{3}(n-5,2)\right)>\cdots>S\left(H_{3}\left(\left\lceil\frac{n-3}{2}\right\rceil,\left\lfloor\frac{n-3}{2}\right\rfloor\right)\right) .
$$

Therefore, our result holds for $p=1,2$.

Next, we assume that $p \geq 3$ and the result holds for all $p^{\prime}$ satisfying that $p^{\prime} \leq p-1$. Note that $V_{p} \cap P V(T) \neq \emptyset$, we assume that $v \in V_{p} \cap P V(T), N_{T}(v)=\{u\}$ and $N_{T}(u)=$ $\left\{v, u_{1}, u_{2}, \ldots, u_{x-1}\right\}$. It is clear that $N_{T}(u) \subseteq V_{p}$, then $d_{T}(u)=x \leq p$. Since $T \neq S_{n}$, there exists at least one neighbour of $u$ with degree greater than two. Without loss of generality, we assume that $d_{T}\left(u_{i}\right) \geq t, i=2,3, \ldots, x-1$. Let $T^{\prime}=T-v$, then $T^{\prime} \in \mathscr{B}_{p-1, q}$. By induction hypothesis, we have $S\left(T^{\prime}\right) \geq S\left(T_{p-1, q}\right)=\sqrt{(p-1)^{2}+q^{2}}+$ $(p-2) \sqrt{1+(p-1)^{2}}+(q-1) \sqrt{1+q^{2}}$. Thus, we have 


$$
\begin{aligned}
S(T)-S\left(T^{\prime}\right)= & \sqrt{x^{2}+1^{2}}+\sum_{i=1}^{x-1}\left[\sqrt{d_{T}\left(u_{i}\right)^{2}+x^{2}}-\sqrt{d_{T}\left(u_{i}\right)^{2}+(x-1)^{2}}\right] \\
= & \sqrt{x^{2}+1^{2}}+(x-2)\left[\sqrt{1+x^{2}}-\sqrt{1+(x-1)^{2}}\right] \\
& +\sqrt{d_{T}\left(u_{1}\right)^{2}+x^{2}}-\sqrt{d_{T}\left(u_{1}\right)^{2}+(x-1)^{2}} \\
= & \sqrt{x^{2}+1^{2}}+(x-2) \phi(x, 1)+\phi\left(x, d_{T}\left(u_{1}\right)\right) \\
\leq & \sqrt{p^{2}+1^{2}}+(p-2) \phi(x, 1)+\phi\left(x, d_{T}\left(u_{1}\right)\right) \\
\leq & \sqrt{p^{2}+1^{2}}+(p-2) \phi(p, 1)+\phi\left(p, d_{T}\left(u_{1}\right)\right) .
\end{aligned}
$$

Moreover, combining with $\sum_{i=1}^{p-1} d\left(u_{i}\right)+d_{T}(v)=p+q-1$ yields that $d_{T}\left(u_{1}\right)=q$. Therefore,

$$
\begin{aligned}
S(T) \leq & S\left(T^{\prime}\right)+\sqrt{p^{2}+1^{2}}+(p-2) \phi(p, 1)+\phi(p, q) \\
\leq & \sqrt{(p-1)^{2}+q^{2}}+(p-2) \sqrt{1+(p-1)^{2}}+(q-1) \sqrt{1+q^{2}} \\
& +\sqrt{p^{2}+1}+(p-2) \phi(p, 1)+\phi(p, q) \\
= & \sqrt{p^{2}+q^{2}}+(p-1) \sqrt{1+p^{2}}+(q-1) \sqrt{1+q^{2}} .
\end{aligned}
$$

The equalities above hold simultaneously if and only if $T^{\prime} \cong S_{p-1, q}, x=p, d_{T}\left(u_{1}\right)=q$ and $d_{T}\left(u_{2}\right)=d_{T}\left(u_{3}\right)=\cdots=d_{T}\left(u_{p-1}\right)=1$, which imply that $T \cong S_{p, q}$.

\subsection{The maximum and second maximum Sombor indices of trees with a given diameter}

Let $\mathscr{D}_{n, d}$ be the set of trees with order $n$ and diameter $d$. We give the maximum and second maximum Sombor indices of trees among $\mathscr{D}_{n, d}$ in this subsection. A tree is said to be a caterpillar if we delete all pendant vertices makes it a path. Let $C_{t_{0}, t_{1}, \ldots, t_{d}}$ be a caterpillar obtained from the path $P=v_{0} v_{1} v_{2} \cdots v_{d}$ by attaching $t_{i}$ edges to the vertex $v_{i}$, $i=0,1,2, \ldots, d-1, d$. Clearly, $T=C_{t_{0}, t_{1}, \ldots, t_{d}}$ belongs to $\mathscr{D}_{n, d}$ if and only if $t_{0}=t_{d}=0$, and the caterpillar $T=C_{t_{0}, t_{1}, \ldots, t_{d}}$ has $n=d+1+\sum_{i=0}^{d} t_{i}$ vertices.

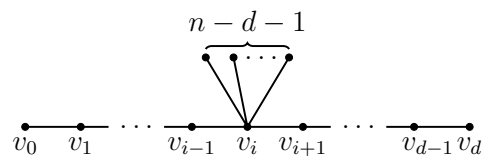

Figure 3. The tree $T_{d}^{i}(n-d-1)$. 
Let $T_{n, d}^{i}$ denote the tree obtained from the path $P=v_{0} v_{1} \ldots v_{d}$ by attaching $n-d-1$ edges to the vertex $v_{i}$, see Figure 3.

Theorem 3.8 Let $T$ be a tree in $\mathscr{D}_{n, d} \backslash\left\{T_{n, d}^{1}\right\}$, then

$$
S(T) \leq 2\left[\sqrt{5}+(d-4) \sqrt{2}+\sqrt{4+(n-d+1)^{2}}\right]+(n-d-1) \sqrt{1+(n-d+1)^{2}}
$$

with equality if and only if $T \cong T_{n, d}^{i}, i=2,3, \ldots,\left\lceil\frac{d}{2}\right\rceil$.

Proof. Let $T \in \mathscr{D}_{n, d} \backslash\left\{T_{n, d}^{1}\right\}$ be the tree with maximum Sombor index and $P=v_{0} v_{1} \ldots v_{d}$ a diametrical path of $T$. We first show that $T$ is a caterpillar. Suppose that $T$ is not a caterpillar, then there exists a non-pendant edge $u v_{i}$, where $u \in V(T) \backslash\left\{v_{0}, v_{1}, \ldots, v_{d}\right\}$. Using the path-lifting transformation on the edge $u v_{i}$, then by Lemma 2.2 we get a new tree $T^{\prime} \in \mathscr{D}_{n, d}$ such that $S\left(T^{\prime}\right)>S(T)$. A contradiction to the choice of $T$. Thus, the tree $T$ can be denoted by $C_{0, t_{1}, \ldots, t_{d-1}, 0}$ and $d_{T}\left(v_{i}\right)=d_{i}, i=0,1, \ldots, d$. Without loss of generality, we always assume that $d_{i} \geq d_{j}$ and $i<j$. There are three special trees which will be used in the following proof, see Figure 4 , where $3 \leq i \leq d-2$ for $T_{2}$. In the following we assume that $T \nRightarrow T_{n, d}^{i}$, then exist two vertex $v_{i}$ and $v_{j}$ such that $d_{T}\left(v_{i}\right) \geq 3$ and $d_{T}\left(v_{j}\right) \geq 3$. We consider the following two possible cases.

Case 1. $v_{i} v_{j} \in E(P)$, i.e., $j=i+1$.

We denote $N_{T}\left(v_{i}\right)=\left\{u_{1}, u_{2}, \ldots, u_{d_{i}-2}, v_{i-1}, v_{j}\right\}, N_{T}\left(v_{j}\right)=\left\{w_{1}, w_{2}, \ldots, w_{d_{j}-2}, v_{i}, v_{j+1}\right\}$, $d_{T}\left(v_{i-1}\right)=x$ and $d_{T}\left(v_{j+1}\right)=y$ in this case.

If $T \cong T_{1}$, then

$$
\begin{aligned}
S\left(T_{1}\right)-S\left(T_{n, d}^{i}\right)= & (n-d-1)\left[\sqrt{1^{2}+(n-d)^{2}}-\sqrt{1^{2}+(n-d+1)^{2}}\right]+\sqrt{3^{2}+(n-d)^{2}} \\
& -\sqrt{2^{2}+(n-d+1)^{2}}+\sqrt{3^{2}+1^{2}}-\sqrt{2^{2}+1^{2}} \\
& -\left(\sqrt{(n-d+1)^{2}+2^{2}}-\sqrt{3^{2}+2^{2}}\right) \\
< & \sqrt{3^{2}+1^{2}}-\sqrt{2^{2}+1^{2}}-\left(\sqrt{(n-d+1)^{2}+2^{2}}-\sqrt{3^{2}+2^{2}}\right) \\
\leq & \sqrt{3^{2}+1^{2}}-\sqrt{2^{2}+1^{2}}-\left(\sqrt{4^{2}+2^{2}}-\sqrt{3^{2}+2^{2}}\right) \quad \text { by } n-d \geq 3 \\
= & \phi(3,1)-\phi(4,2)=-2 \sqrt{5}+\sqrt{10}+\sqrt{13}-3 \approx-0.704307<0 .
\end{aligned}
$$

A contradiction to the choice of $T$. Thus, $T \neq T_{1}$.

Let $T^{\prime}=T-v_{j} w_{1}+v_{i} w_{1}$. Then, $T^{\prime} \in \mathscr{D}_{n, d} \backslash\left\{T_{n, d}^{1}\right\}, d_{T^{\prime}}\left(v_{i}\right)=d_{i}+1, d_{T^{\prime}}=d_{j}-1$ and

$$
S\left(T^{\prime}\right)-S(T)=\sqrt{x^{2}+\left(d_{i}+1\right)^{2}}+\left(d_{i}-1\right) \sqrt{1^{2}+\left(d_{i}+1\right)^{2}}+\left(d_{j}-3\right) \sqrt{1^{2}+\left(d_{j}-1\right)^{2}}
$$




$$
\begin{aligned}
& +\sqrt{y^{2}+\left(d_{j}-1\right)^{2}}-\sqrt{x^{2}+d_{i}^{2}}-\left(d_{i}-2\right) \sqrt{1^{2}+d_{i}^{2}} \\
& -\left(d_{j}-2\right) \sqrt{1^{2}+d_{j}^{2}}-\sqrt{y^{2}+d_{j}^{2}} \\
= & \phi\left(d_{i}+1, x\right)-\phi\left(d_{j}, y\right)+\left(d_{i}-2\right) \phi\left(d_{i}+1,1\right)-\left(d_{j}-3\right) \phi\left(d_{j}, 1\right) \\
& +\sqrt{1^{2}+\left(d_{i}+1\right)^{2}}-\sqrt{1^{2}+d_{j}^{2}} \\
\geq & \phi\left(d_{i}+1, x\right)-\phi\left(d_{j}, y\right)+\left(d_{i}-2\right) \phi\left(d_{i}+1,1\right)-\left(d_{j}-3\right) \phi\left(d_{j}, 1\right) \\
& +\sqrt{1^{2}+\left(d_{j}+1\right)^{2}}-\sqrt{1^{2}+d_{j}^{2}} \\
= & \phi\left(d_{i}+1, x\right)-\phi\left(d_{j}, y\right)+\left(d_{i}-2\right) \phi\left(d_{i}+1,1\right)-\left(d_{j}-3\right) \phi\left(d_{j}, 1\right) \\
+ & \phi\left(d_{j}+1,1\right)>\phi\left(d_{i}+1, x\right)-\phi\left(d_{j}, y\right)+\left(d_{j}-3\right)\left[\phi\left(d_{i}+1,1\right)-\phi\left(d_{j}, 1\right)\right] \\
+ & \phi\left(d_{j}+1,1\right)>\phi\left(d_{j}+1,1\right)-\phi\left(d_{j}, y\right) \geq \phi\left(d_{j}+1,1\right)-\phi\left(d_{j}, 1\right)>0 .
\end{aligned}
$$

We get a contradiction that $S\left(T^{\prime}\right)>S(T)$.

Case 2. $v_{i} v_{j} \notin E(P)$, i.e., $j \geq i+2$.

We denote $N_{T}\left(v_{i}\right)=\left\{u_{1}, u_{2}, \ldots, u_{d_{i}-2}, v_{i-1}, v_{i+1}\right\}, N_{T}\left(v_{j}\right)=\left\{w_{1}, \ldots, w_{d_{j}-2}, v_{j-1}\right.$, $\left.v_{j+1}\right\}, d_{T}\left(v_{i-1}\right)=x$ and $d_{T}\left(v_{j+1}\right)=y$ in this case.

If $T \cong T_{2}$ or $T \cong T_{3}$, then we can get that

$$
\begin{aligned}
S\left(T_{2}\right)-S\left(T_{n, d}^{i}\right)= & (n-d-1)\left[\sqrt{1^{2}+(n-d)^{2}}-\sqrt{1^{2}+(n-d+1)^{2}}\right] \\
& +\sqrt{2^{2}+(n-d)^{2}}-\sqrt{2^{2}+(n-d+1)^{2}}+\sqrt{3^{2}+1^{2}}-\sqrt{2^{2}+1^{2}} \\
& -\left(\sqrt{(n-d+1)^{2}+2^{2}}-\sqrt{3^{2}+2^{2}}\right) \\
< & \sqrt{3^{2}+1^{2}}-\sqrt{2^{2}+1^{2}}-\left(\sqrt{(n-d+1)^{2}+2^{2}}-\sqrt{3^{2}+2^{2}}\right) \\
\leq & \sqrt{3^{2}+1^{2}}-\sqrt{2^{2}+1^{2}}-\left(\sqrt{4^{2}+2^{2}}-\sqrt{3^{2}+2^{2}}\right) \quad \text { since } n-d \geq 3 \\
= & \phi(3,1)-\phi(4,2)=-2 \sqrt{5}+\sqrt{10}+\sqrt{13}-3 \approx-0.704307<0
\end{aligned}
$$

and

$$
\begin{aligned}
S\left(T_{3}\right)-S\left(T_{n, d}^{i}\right)= & (n-d-1)\left[\sqrt{1^{2}+(n-d)^{2}}-\sqrt{1^{2}+(n-d+1)^{2}}\right] \\
& +\sqrt{2^{2}+(n-d)^{2}}-\sqrt{2^{2}+(n-d+1)^{2}} \\
& +2\left(\sqrt{3^{2}+1^{2}}-\sqrt{2^{2}+1^{2}}\right)-\left(\sqrt{(n-d+1)^{2}+2^{2}}-\sqrt{3^{2}+2^{2}}\right) \\
< & 2\left(\sqrt{3^{2}+1^{2}}-\sqrt{2^{2}+1^{2}}\right)-\left(\sqrt{(n-d+1)^{2}+2^{2}}-\sqrt{3^{2}+2^{2}}\right) \\
< & 2\left(\sqrt{3^{2}+1^{2}}-\sqrt{2^{2}+1^{2}}\right)-2\left(\sqrt{4^{2}+2^{2}}-\sqrt{3^{2}+2^{2}}\right) \quad \text { by } n-d \geq 3 \\
= & 2 \phi(3,1)-2 \phi(4,2)=-4 \sqrt{5}+2 \sqrt{10}+2 \sqrt{13}-6 \approx-1.40861<0 .
\end{aligned}
$$

A contradiction to the choice of $T$. Thus, $T \nRightarrow T_{2}$ and $T ¥ T_{3}$. 
Let $T^{\prime \prime}=T-v_{j} w_{1}+v_{i} w_{1}$. Then, $T^{\prime \prime} \in \mathscr{D}_{n, d} \backslash\left\{T_{n, d}^{1}\right\}, d_{T^{\prime \prime}}\left(v_{i}\right)=d_{i}+1, d_{T^{\prime \prime}}\left(v_{j}\right)=d_{j}-1$ and

$$
\begin{aligned}
S\left(T^{\prime \prime}\right)-S(T)= & \sqrt{x^{2}+\left(d_{i}+1\right)^{2}}+\left(d_{i}-1\right) \sqrt{1^{2}+\left(d_{i}+1\right)^{2}}+\left(d_{j}-3\right) \sqrt{1^{2}+\left(d_{j}-1\right)^{2}} \\
& +\sqrt{y^{2}+\left(d_{j}-1\right)^{2}}+\sqrt{2^{2}+\left(d_{i}+1\right)^{2}}+\sqrt{2^{2}+\left(d_{j}-1\right)^{2}} \\
& -\sqrt{x^{2}+d_{i}^{2}}-\left(d_{i}-2\right) \sqrt{1^{2}+d_{i}^{2}}-\left(d_{j}-2\right) \sqrt{1^{2}+d_{j}^{2}} \\
& -\sqrt{y^{2}+d_{j}^{2}}-\sqrt{2^{2}+d_{i}^{2}}-\sqrt{2^{2}+d_{j}^{2}} \\
= & \phi\left(d_{i}+1, x\right)-\phi\left(d_{j}, y\right)+\left(d_{i}-2\right) \phi\left(d_{i}+1,1\right)-\left(d_{j}-3\right) \phi\left(d_{j}, 1\right) \\
& +\phi\left(d_{i}+1,2\right)-\phi\left(d_{j}, 2\right)+\sqrt{1^{2}+\left(d_{i}+1\right)^{2}}-\sqrt{1^{2}+d_{j}^{2}} \\
> & \phi\left(d_{i}+1, x\right)-\phi\left(d_{j}, y\right)+\left(d_{i}-2\right) \phi\left(d_{i}+1,1\right)-\left(d_{j}-3\right) \phi\left(d_{j}, 1\right) \\
& +\sqrt{1^{2}+\left(d_{j}+1\right)^{2}}-\sqrt{1^{2}+d_{j}^{2}} \\
= & \phi\left(d_{i}+1, x\right)-\phi\left(d_{j}, y\right)+\left(d_{i}-2\right) \phi\left(d_{i}+1,1\right)-\left(d_{j}-3\right) \phi\left(d_{j}, 1\right) \\
+ & \phi\left(d_{j}+1,1\right)>\phi\left(d_{i}+1, x\right)-\phi\left(d_{j}, y\right)+\left(d_{j}-3\right)\left[\phi\left(d_{i}+1,1\right)-\phi\left(d_{j}, 1\right)\right] \\
+ & \phi\left(d_{j}+1,1\right)>\phi\left(d_{j}+1,1\right)-\phi\left(d_{j}, y\right) \geq \phi\left(d_{j}+1,1\right)-\phi\left(d_{j}, 1\right)>0 .
\end{aligned}
$$

We get a contradiction that $S\left(T^{\prime \prime}\right)>S(T)$.

Therefore, from the discussion above we conclude that $T$ is a caterpillar with only one vertex $v_{i}$ on the diametrical path such that $d_{T}\left(v_{i}\right) \geq 3$. Note that $T \neq T_{n, d}^{1}$, then $T \cong T_{n, d}^{i}, i=2,3, \ldots,\left\lceil\frac{d}{2}\right\rceil$.
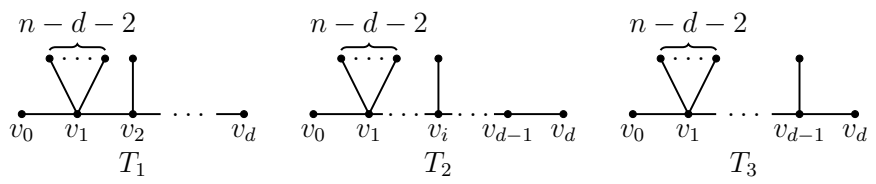

Figure 4. The trees $T_{1}, T_{2}$ and $T_{3}$ in the proof of Theorem 3.8 .

Theorem 3.9 Let $T$ be a tree in $\mathscr{D}_{n, d}$, then

$$
S(T) \leq(n-d) \sqrt{(n-d+1)^{2}+1}+\sqrt{(n-d+1)^{2}+4}+2(d-3) \sqrt{2}+\sqrt{5},
$$

with equality if and only if $T \cong T_{n, d}^{1}$.

Proof. For $i=2,3, \ldots,\left\lceil\frac{d}{2}\right\rceil$, by direct calculation, we have

$$
S\left(T_{n, n-d-1}^{1}\right)-S\left(T_{n, n-d-1}^{i}\right)=\phi(2,2)-\phi(2, n-d+1)>0
$$


Thus, the result follows immediately by Theorem 3.8 .

Theorems 3.8 and 3.9 show that among all trees of order $n$ with diameter $d$ the tree $T_{n, d}^{1}$ possesses the maximum Sombor index and the trees which attain the second maximum Sombor index belong to $\left\{T_{n, d}^{i}: i=2,3, \ldots,\left\lceil\frac{d}{2}\right\rceil\right\}$.

\subsection{The minimum and maximum Sombor index of trees with given number of segments}

A segment of a tree is a path-subtree whose terminal vertices are branching or pendant vertices. The so-called starlike tree is a tree contains exactly one vertex of degree greater than two. Let $\mathscr{F}_{n, k}$ be the set of trees of order $n$ with exactly $k$ segments. Then it is easy to see that the path $P_{n}$ is the unique graph in $\mathscr{F}_{n, 1}$ and the set $\mathscr{F}_{n, 2}=\emptyset$. Thus, we only need to consider $\mathscr{F}_{n, k}$ for $3 \leq k \leq n-2$ in this subsection.

Lemma 3.10 If $T$ is a tree in $\mathscr{F}_{n, k}$ with minimal Sombor index, then $T$ must be a chemical tree, i.e., the tree does not contain a vertex of degree greater than four.

Proof. Suppose to the contrary that there is a vertex $v$ with $d_{T}(v) \geq 5$. Let $P=$ $v_{0} v_{1} \ldots v_{l} v_{l+1}$ be a longest path in $T$ containing $v=v_{i}$. Then one can pick two vertices $u$ and $w$ such that $u, w \in N_{T}(v) \backslash V(P)$.

Case 1. $i=l$ or $i=1$.

Then the vertices in $N_{T}(v) \backslash\left\{v_{2}\right\}$ or $N_{T}(v) \backslash\left\{v_{l-1}\right\}$ are all pendant vertices in this case. We only consider the case of $i=1$ and the the case of $i=l$ can be proved analogously. Let $T^{\prime}=T-\{v u, v w\}+\left\{v_{0} u, v_{0} w\right\}$. It is obvious that $T^{\prime}$ also belongs to $\mathscr{F}_{n, k}$. One can see that $d_{T^{\prime}}(v)=d_{T}(v)-2, d_{T^{\prime}}\left(v_{0}\right)=3$ and the degrees of the rest vertices are unchanged. Thus we have

$$
\begin{aligned}
S(T)-S\left(T^{\prime}\right)= & \left(d_{T}\left(v_{1}\right)-1\right) \sqrt{d_{T}\left(v_{1}\right)^{2}+1^{2}}+\sqrt{d_{T}\left(v_{1}\right)^{2}+d_{T}\left(v_{2}\right)^{2}} \\
& -\left(d_{T}\left(v_{1}\right)-4\right) \sqrt{\left(d_{T}\left(v_{1}\right)-2\right)^{2}+1^{2}}-\sqrt{\left(d_{T}\left(v_{1}\right)-2\right)^{2}+d_{T}\left(v_{2}\right)^{2}} \\
& -\sqrt{d_{T}\left(v_{1}\right)^{2}+3^{2}}-\sqrt{1^{2}+3^{3}}-\sqrt{1^{2}+3^{3}} \\
> & 3 \sqrt{d_{T}\left(v_{1}\right)^{2}+1^{2}}-\sqrt{d_{T}\left(v_{1}\right)^{2}+3^{2}}-2 \sqrt{10} \\
\geq & 3 \sqrt{5^{2}+1^{2}}-\sqrt{5^{2}+3^{2}}-2 \sqrt{10} \quad \text { since } d_{T}\left(v_{1}\right)=d_{T}(v) \geq 5 \\
\approx & 3.1416>0,
\end{aligned}
$$

a contradiction. 
Case 2. $2 \leq i \leq l-1$.

By previous case it holds that $d_{T}\left(v_{1}\right) \leq 4$ and $d_{T}\left(v_{l}\right) \leq 4$. Without loss of generality, we suppose that $d_{T}\left(v_{i}\right)=\max \left\{d_{T}(w): w \in V(T)\right\} \geq 5$. Let $T^{\prime}=T-\left\{v_{i} u, v_{i} w\right\}+$ $\left\{v_{l+1} u, v_{l+1} w\right\}$. Since for any vertex $z \in N\left(v_{i}\right) \backslash\{u, w\}$ we have $d_{T}\left(v_{i}\right) \geq d_{T}(z)$, then it is easy to verify that $\sqrt{d_{T}\left(v_{i}\right)^{2}+d_{T}(z)^{2}}-\sqrt{\left(d_{T}\left(v_{i}\right)-2\right)^{2}+d_{T}(z)^{2}}=\phi\left(d_{T}\left(v_{i}\right), d_{T}(z)\right)+$ $\phi\left(d_{T}\left(v_{i}\right)-1, d_{T}(z)\right) \geq \phi(5,5)+\phi(4,5)$ and $\sqrt{d_{T}\left(v_{l}\right)^{2}+1^{2}}-\sqrt{3^{2}+d_{T}\left(v_{l}\right)^{2}} \geq \sqrt{4^{2}+1^{2}}-$ $\sqrt{3^{2}+4^{2}}$. So, we have

$$
\begin{aligned}
S(T)-S\left(T^{\prime}\right) & =\sqrt{d_{T}\left(v_{i}\right)^{2}+d_{T}(u)^{2}}+\sqrt{d_{T}\left(v_{i}\right)^{2}+d_{T}(w)^{2}} \\
& +\sum_{z \in N\left(v_{i}\right) \backslash\{u, w\}} \sqrt{d_{T}\left(v_{i}\right)^{2}+d_{T}(z)^{2}} \\
& +\sqrt{d_{T}\left(v_{l}\right)^{2}+1^{2}}-\sqrt{3^{2}+d_{T}(u)^{2}} \\
& -\sqrt{3^{2}+d_{T}(w)^{2}}-\sum_{z \in N\left(v_{i}\right) \backslash\{u, w\}} \sqrt{\left(d_{T}\left(v_{i}\right)-2\right)^{2}+d_{T}(z)^{2}}-\sqrt{3^{2}+d_{T}\left(v_{l}\right)^{2}} \\
& >\sum_{z \in N\left(v_{i}\right) \backslash\{u, w\}}\left[\sqrt{d_{T}\left(v_{i}\right)^{2}+d_{T}(z)^{2}}-\sqrt{\left(d_{T}\left(v_{i}\right)-2\right)^{2}+d_{T}(z)^{2}}\right] \\
& +\sqrt{d_{T}\left(v_{l}\right)^{2}+1^{2}}-\sqrt{3^{2}+d_{T}\left(v_{l}\right)^{2}}>3[\phi(5,5)+\phi(4,5)]+\sqrt{4^{2}+1^{2}} \\
& -\sqrt{3^{2}+4^{2}}=15 \sqrt{2}-3 \sqrt{34}+\sqrt{17}-5 \approx 2.8435>0 .
\end{aligned}
$$

Thus, a contradiction to the choice of $T$. The proof is completed.

Lemma 3.11 If $T$ is a tree in $\mathscr{F}_{n, k}$ with minimal Sombor index, then $T$ contain at most one vertex of degree four.

Proof. From Lemma 3.10 we know that $\Delta(T) \leq 4$. Now, we suppose to the contrary that there are at least two vertices $v_{i}$ and $v_{j}$ having degree four in $T$ and let $P=v_{0} v_{1} \ldots v_{l} v_{l+1}$ be a longest path in $T$ containing $v_{i}$ and $v_{j}$. Then one can pick two vertices $u$ and $w$ such that $\{u, w\} \nsubseteq V(P)$ and $\left\{u v_{i}, w v_{j}\right\} \subseteq E(T)$. Let $T^{\prime}=T-\left\{u v_{i}, w v_{j}\right\}+\left\{u v_{l+1}, w v_{l+1}\right\}$. Then we can see that $T^{\prime}$ also belongs to $\mathscr{F}_{n, k}$. Now, we consider the following four cases respectively.

Case 1. $l=j$ and $v_{i} v_{j} \notin E(T)$.

$$
\begin{aligned}
S(T)-S\left(T^{\prime}\right)= & \sqrt{4^{2}+d_{T}(u)^{2}}+\sqrt{4^{2}+d_{T}(w)^{2}}+\sum_{y \in N\left(v_{i}\right) \backslash\{u\}} \sqrt{4^{2}+d_{T}(y)^{2}} \\
& +\sum_{z \in N\left(v_{j}\right) \backslash\left\{w, v_{l+1}\right\}} \sqrt{4^{2}+d_{T}(z)^{2}}+\sqrt{4^{2}+1^{2}}-\sqrt{3^{2}+d_{T}(u)^{2}} \\
& -\sqrt{3^{2}-d_{T}(w)^{2}}-\sum_{y \in N\left(v_{i}\right) \backslash\{u\}} \sqrt{3^{2}+d_{T}(y)^{2}}
\end{aligned}
$$




$$
\begin{aligned}
& -\sum_{z \in N\left(v_{j}\right) \backslash\left\{w, v_{l+1}\right\}} \sqrt{3^{2}+d_{T}(z)^{2}}-\sqrt{3^{2}+3^{2}} \\
> & \sum_{y \in N\left(v_{i}\right) \backslash\{u\}}\left(\sqrt{4^{2}+d_{T}(y)^{2}}-\sqrt{3^{2}+d_{T}(y)^{2}}\right)+\sqrt{4^{2}+1^{2}}-\sqrt{3^{2}+3^{2}} \\
& +\sum_{z \in N\left(v_{j}\right) \backslash\left\{w, v_{l+1}\right\}}\left(\sqrt{4^{2}+d_{T}(z)^{2}}-\sqrt{3^{2}+d_{T}(z)^{2}}\right) \\
\geq & 3 \phi(4,4)+2 \phi(4,4)+\sqrt{4^{2}+1^{2}}-\sqrt{3^{2}+3^{2}} \\
= & 5(4 \sqrt{2}-5)+\sqrt{17}-3 \sqrt{2} \approx 3.1647>0,
\end{aligned}
$$

a contradiction.

Case 2. $l \neq j$ and $v_{i} v_{j} \notin E(T)$.

$$
\begin{aligned}
S(T)-S\left(T^{\prime}\right)= & \sqrt{4^{2}+d_{T}(u)^{2}}+\sqrt{4^{2}+d_{T}(w)^{2}}+\sum_{y \in N\left(v_{i}\right) \backslash\{u\}} \sqrt{4^{2}+d_{T}(y)^{2}} \\
& +\sum_{z \in N\left(v_{j}\right) \backslash\{w\}} \sqrt{4^{2}+d_{T}(z)^{2}}+\sqrt{d_{T}\left(v_{l}\right)^{2}+1^{2}}-\sqrt{3^{2}+d_{T}(u)^{2}} \\
& -\sqrt{3^{2}-d_{T}(w)^{2}}-\sum_{y \in N\left(v_{i}\right) \backslash\{u\}} \sqrt{3^{2}+d_{T}(y)^{2}} \\
& -\sum_{z \in N\left(v_{j}\right) \backslash\{w\}} \sqrt{3^{2}+d_{T}(z)^{2}}-\sqrt{d_{T}\left(v_{l}\right)^{2}+3^{2}} \\
> & \sum_{y \in N\left(v_{i}\right) \backslash\{u\}}\left(\sqrt{4^{2}+d_{T}(y)^{2}}-\sqrt{3^{2}+d_{T}(y)^{2}}\right)+\sqrt{d_{T}\left(v_{l}\right)^{2}+1^{2}} \\
& -\sqrt{d_{T}\left(v_{l}\right)^{2}+3^{2}}+\sum_{z \in N\left(v_{j}\right) \backslash\{w\}}\left(\sqrt{4^{2}+d_{T}(z)^{2}}-\sqrt{3^{2}+d_{T}(z)^{2}}\right) \\
\geq & 3 \phi(4,4)+2 \phi(4,4)+\sqrt{2^{2}+1^{2}}-\sqrt{2^{2}+3^{2}} \\
= & 5(4 \sqrt{2}-5)+\sqrt{5}-\sqrt{13} \approx 1.9148>0,
\end{aligned}
$$

a contradiction.

Case 3. $l \neq j$ and $v_{i} v_{j} \in E(T)$.

$$
\begin{aligned}
S(T)-S\left(T^{\prime}\right)= & \sqrt{4^{2}+d_{T}(u)^{2}}+\sqrt{4^{2}+d_{T}(w)^{2}}+\sqrt{4^{2}+4^{2}}+\sum_{y \in N\left(v_{i}\right) \backslash\left\{u, v_{j}\right\}} \sqrt{4^{2}+d_{T}(y)^{2}} \\
& +\sum_{z \in N\left(v_{j}\right) \backslash\left\{w, v_{i}\right\}} \sqrt{4^{2}+d_{T}(z)^{2}}+\sqrt{d_{T}\left(v_{l}\right)^{2}+1^{2}}-\sqrt{3^{2}+d_{T}(u)^{2}} \\
& -\sqrt{3^{2}-d_{T}(w)^{2}}-\sum_{y \in N\left(v_{i}\right) \backslash\left\{u, v_{j}\right\}} \sqrt{3^{2}+d_{T}(y)^{2}} \\
& -\sum_{z \in N\left(v_{j}\right) \backslash\left\{w, v_{i}\right\}} \sqrt{3^{2}+d_{T}(z)^{2}}-\sqrt{d_{T}\left(v_{l}\right)^{2}+3^{2}}-\sqrt{3^{2}+3^{2}} \\
> & \sum_{y \in N\left(v_{i}\right) \backslash\left\{u, v_{j}\right\}}\left(\sqrt{4^{2}+d_{T}(y)^{2}}-\sqrt{3^{2}+d_{T}(y)^{2}}\right)+\sqrt{d_{T}\left(v_{l}\right)^{2}+1^{2}}
\end{aligned}
$$




$$
\begin{aligned}
& -\sqrt{d_{T}\left(v_{l}\right)^{2}+3^{2}}+\sum_{z \in N\left(v_{j}\right) \backslash\left\{w, v_{i}\right\}}\left(\sqrt{4^{2}+d_{T}(z)^{2}}-\sqrt{3^{2}+d_{T}(z)^{2}}\right) \\
\geq & 2 \phi(4,4)+2 \phi(4,4)+\sqrt{2^{2}+1^{2}}-\sqrt{2^{2}+3^{2}} \\
= & 16 \sqrt{2}+\sqrt{5}-\sqrt{13}>0,
\end{aligned}
$$

a contradiction.

Case 4. $l=j$ and $v_{i} v_{j} \in E(T)$. Let $N_{T}\left(v_{i}\right)=\left\{u, v_{j}, v_{i-1}, z\right\}$ and note that, in this case, the vertex $v_{j}$ has three pendant vertices as neighbors. Thus we have

$$
\begin{aligned}
S(T)-S\left(T^{\prime}\right)= & \sqrt{4^{2}+d_{T}(u)^{2}}+\sqrt{4^{2}+d_{T}(z)^{2}}+\sqrt{4^{2}+d_{T}\left(v_{i-1}\right)^{2}}+\sqrt{4^{2}+4^{2}} \\
& +3 \sqrt{1^{2}+4^{2}}-\sqrt{3^{2}+d_{T}(u)^{2}}-\sqrt{3^{2}+d_{T}(z)^{2}} \\
& -\sqrt{3^{2}+d_{T}\left(v_{i-1}\right)^{2}}-2 \sqrt{3^{2}+3^{2}}-2 \sqrt{1^{2}+3^{2}} \\
> & \sqrt{4^{2}+4^{2}}+3 \sqrt{1^{2}+4^{2}}-2 \sqrt{3^{2}+3^{2}}-2 \sqrt{1^{2}+3^{2}} \\
= & 3 \sqrt{17}-2 \sqrt{2}-2 \sqrt{10} \approx 3.2163>0,
\end{aligned}
$$

a contradiction.

Therefore, combining the four cases above we can conclude that the tree with minimal Sombor index contains at most one vertex of degree four.

Using a similar method previously used by Borovićanin (see Lemma 2.3 in [4] for detail), we can obtain that

Lemma 3.12 Let $T$ be a tree in $\mathscr{F}_{n, k}$ with minimal Sombor index. If $n_{4}=0$, then $n_{1}=n_{3}+2$ and $k$ is odd. If $n_{4}=1$, then $n_{1}=n_{3}+4$ and $k$ is even. Moreover, if $n_{4}=0$ then $n_{1}=\frac{k+3}{2}, n_{2}=n-k-1$ and $n_{3}=\frac{k-1}{2}$; if $n_{4}=1$ then $n_{1}=\frac{k+4}{2}, n_{2}=n-k-1$ and $n_{3}=\frac{k-4}{2}$.

Lemma 3.13 (i) Let $P=v_{1} v_{2} \ldots u_{t}$ be a segment of a tree $T \in \mathscr{F}_{n, k}$ such that $d_{T}\left(v_{1}\right)=$ $3, d_{T}\left(v_{t}\right)=3$ or $d_{T}\left(v_{t}\right)=4$, where $t \geq 3$. Let $u_{1}$ be a pendant vertex of $T$ and $u_{2}$ the unique neighbor of $u_{1}$, satisfying $d_{T}\left(u_{2}\right)=2$ or $d_{T}\left(u_{2}\right)=3$ (here, $u_{2}$ may coincide with $\left.v_{1}\right)$. $T^{\prime}=T-v_{1} v_{2}-v_{t-1} v_{t}+v_{t-1} u_{1}+v_{1} v_{t}$. Then we have $S(T)>S\left(T^{\prime}\right)$.

(ii) Let $v_{1}, v_{2}, u_{1}, u_{2}$ be the vertices of a tree in $\mathscr{F}_{n, k}$ such that $v_{1} v_{2}, u_{1} u_{2} \in E(T)$, $d_{T}\left(v_{1}\right)=d_{T}\left(v_{2}\right)=3, d_{T}\left(u_{1}\right)=4$ and $d_{T}\left(u_{2}\right)=2$ or $d_{T}\left(u_{2}\right)=1$. Let $T^{\prime}$ be obtained form $T$ by deleting edges $v_{1} v_{2}, u_{1} u_{2}$ and then adding edges $v_{1} u_{2}, u_{1} v_{2}$, i.e. $T^{\prime}=T-v_{1} v_{2}-u_{1} u_{2}+v_{1} u_{2}+u_{1} v_{2}$. Then we have $S(T)>S\left(T^{\prime}\right)$. 
(iii) Let $v_{1}, v_{2}, v_{3}, u_{1}, u_{2}$ be the vertices of a tree $T$ in $\mathscr{F}_{n, k}$ such that $v_{1} v_{2}, v_{2} v_{3}, u_{1} u_{2} \in$ $E(T), d_{T}\left(v_{1}\right)=d_{T}\left(v_{2}\right)=2, d_{T}\left(v_{3}\right)=3, d_{T}\left(u_{1}\right)=1, d_{T}\left(u_{2}\right)=3$ or $d_{T}\left(u_{2}\right)=4$. Let $T^{\prime}=T-v_{1} v_{2}-v_{2} v_{3}+u_{1} v_{2}+v_{1} v_{3}$. Then we have $S(T)>S\left(T^{\prime}\right)$.

Proof. (i) If $d_{T}\left(v_{t}\right)=3$ and $d_{T}\left(u_{2}\right)=2$, then it holds that

$$
S(T)-S\left(T^{\prime}\right)=\sqrt{2^{2}+3^{2}}+\sqrt{2^{2}+3^{2}}-\sqrt{3^{2}+3^{2}}-\sqrt{2^{2}+2^{2}} \approx 0.140035>0 .
$$

If $d_{T}\left(v_{t}\right)=3$ and $d_{T}\left(u_{2}\right)=3$, then it holds that

$$
S(T)-S\left(T^{\prime}\right)=\sqrt{2^{2}+3^{2}}+\sqrt{1^{2}+3^{2}}-\sqrt{3^{2}+3^{2}}-\sqrt{1^{2}+2^{2}} \approx 0.28912>0 .
$$

If $d_{T}\left(v_{t}\right)=4$ and $d_{T}\left(u_{2}\right)=2$, then it holds that

$$
S(T)-S\left(T^{\prime}\right)=\sqrt{2^{2}+3^{2}}+\sqrt{4^{2}+2^{2}}-\sqrt{2^{2}+2^{2}}-\sqrt{3^{2}+4^{2}} \approx 0.24926>0 .
$$

If $d_{T}\left(v_{t}\right)=4$ and $d_{T}\left(u_{2}\right)=3$, then it holds that

$$
S(T)-S\left(T^{\prime}\right)=\sqrt{1^{2}+3^{2}}+\sqrt{4^{2}+2^{2}}-\sqrt{1^{2}+2^{2}}-\sqrt{3^{2}+4^{2}} \approx 0.39846>0 .
$$

(ii) If $d_{T}\left(v_{1}\right)=d_{T}\left(v_{2}\right)=3, d_{T}\left(u_{1}\right)=4$ and $d_{T}\left(u_{2}\right)=2$, then

$$
S(T)-S\left(T^{\prime}\right)=\sqrt{3^{2}+3^{2}}+\sqrt{4^{2}+2^{2}}-\sqrt{3^{2}+2^{2}}-\sqrt{3^{2}+4^{2}} \approx 0.109225>0 .
$$

If $d_{T}\left(v_{1}\right)=d_{T}\left(v_{2}\right)=3, d_{T}\left(u_{1}\right)=4$ and $d_{T}\left(u_{2}\right)=1$, then

$$
S(T)-S\left(T^{\prime}\right)=\sqrt{3^{2}+3^{2}}+\sqrt{4^{2}+1^{2}}-\sqrt{3^{2}+1^{2}}-\sqrt{3^{2}+4^{2}} \approx 0.203469>0 .
$$

(iii) If $d_{T}\left(v_{1}\right)=d_{T}\left(v_{2}\right)=2, d_{T}\left(u_{1}\right)=1$ and $d_{T}\left(v_{3}\right)=d_{T}\left(u_{2}\right)=3$, then

$$
S(T)-S\left(T^{\prime}\right)=\sqrt{1^{2}+3^{2}}+\sqrt{2^{2}+2^{2}}-\sqrt{2^{2}+3^{2}}-\sqrt{1^{2}+2^{2}} \approx 0.149086>0 .
$$

If $d_{T}\left(v_{1}\right)=d_{T}\left(v_{2}\right)=2, d_{T}\left(u_{1}\right)=1, d_{T}\left(v_{3}\right)=3$ and $d_{T}\left(u_{2}\right)=4$, then

$$
S(T)-S\left(T^{\prime}\right)=\sqrt{1^{2}+4^{2}}+\sqrt{2^{2}+2^{2}}-\sqrt{2^{2}+4^{2}}-\sqrt{1^{2}+2^{2}} \approx 0.243329>0 .
$$

Thus, we are done.

Let $\pi_{1}=(\underbrace{3,3, \ldots, 3}_{\frac{k-1}{2}}, \underbrace{2,2, \ldots, 2}_{n-k-1}, \underbrace{1,1, \ldots, 1}_{\frac{k+3}{2}})$ and $\pi_{2}=(4, \underbrace{3,3, \ldots, 3}_{\frac{k-4}{2}}, \underbrace{2,2, \ldots, 2}_{n-k-1}$, $\underbrace{1,1, \ldots, 1}_{\frac{k+4}{2}}$. Now let us define some families of trees which will be used later. Let $\mathcal{F}_{n, k}^{1}$ denote the set of trees with degree sequence $\pi_{1}$ and $m_{3,3}=\frac{k-3}{2}, m_{2,3}=\frac{k+3}{2}, m_{1,2}=\frac{k+3}{2}$, 
$m_{2,2}=\frac{2 n-3 k-5}{2}$ for $3 \leq k \leq \frac{2 n-5}{3}$ and $k$ is odd. Let $\mathcal{F}_{n, k}^{2}$ be the set of trees with degree sequence $\pi_{1}$ and $m_{3,3}=\frac{k-3}{2}, m_{2,3}=n-k-1, m_{1,2}=n-k-1, m_{1,3}=\frac{3 k-2 n+5}{2}$ for $\frac{2 n-5}{3}<k \leq n-2$ and $k$ is odd. Let $\mathcal{F}_{n, k}^{3}$ be the set of trees with degree sequence $\pi_{2}$ and $m_{1,2}=\frac{k+4}{2}, m_{2,2}=n-\frac{3 k}{2}-3, m_{2,3}=k-4, m_{2,4}=\frac{12-k}{2}, m_{3,4}=\frac{k-4}{2}$ for $4 \leq k \leq 10$ and $k$ is even. Let $\mathcal{F}_{n, k}^{4}$ be the set of trees with degree sequence $\pi_{2}$ and $m_{1,2}=\frac{k+4}{2}$, $m_{2,2}=n-\frac{3}{2} k-3, m_{2,3}=\frac{k+4}{2}, m_{3,3}=\frac{k-12}{2}, m_{3,4}=4$ for $12 \leq k \leq \frac{2 n-6}{3}$ and $k$ is even. Let $\mathcal{F}_{n, k}^{5}$ be the set of trees with degree sequence $\pi_{2}$ and $m_{1,2}=n-k-1, m_{1,3}=\frac{3}{2} k-n+3$, $m_{2,3}=n-k-1, m_{3,3}=\frac{k-12}{2}, m_{3,4}=4$ for $k>\frac{2 n-6}{3}$ and $k$ is even.

Theorem 3.14 Let $T$ be a tree in $\mathscr{F}_{n, k}$.

(i) If $3 \leq k \leq \frac{2 n-5}{3}$ and $k$ is odd, then $S(T) \geq \frac{3(k-3) \sqrt{2}+(k+3)(\sqrt{13}+\sqrt{5})}{2}+(2 n-3 k-5) \sqrt{2}$ with equality if and only if $T \in \mathcal{F}_{n, k}^{1}$.

(ii) If $\frac{2 n-5}{3}<k \leq n-2$ and $k$ is odd, then $S(T) \geq \frac{3(k-3) \sqrt{2}+(3 k-2 n+5) \sqrt{10}}{2}+(n-k-$ 1) $(\sqrt{13}+\sqrt{5})$ with equality if and only if $T \in \mathcal{F}_{n, k}^{2}$.

(iii) If $4 \leq k \leq 10$ and $k$ is even, then $S(T) \geq \frac{(28-k) \sqrt{5}+2 \sqrt{13}+5 k-20}{2}+(2 n-3 k-6) \sqrt{2}$ with equality if and only if $T \in \mathcal{F}_{n, k}^{3}$.

(iv) If $12 \leq k \leq \frac{2 n-6}{3}$ and $k$ is even, then $S(T) \geq \frac{(\sqrt{5}+\sqrt{13})(k+4)+3 \sqrt{2}(k-12)}{2}+\sqrt{2}(2 n-3 k-$ $6)+20$ with equality if and only if $T \in \mathcal{F}_{n, k}^{4}$.

(v) If $\frac{2 n-6}{3}<k \leq n-2$ and $k$ is even, then $S(T) \geq(\sqrt{5}+\sqrt{13})(n-k-1)+\frac{\sqrt{10}}{2}(3 k-$ $2 n+6)+\frac{3 \sqrt{2}}{2}(k-12)+20$ with equality if and only if $T \in \mathcal{F}_{n, k}^{5}$.

Proof. Let $T$ be a tree in $\mathscr{F}_{n, k}$ with minimal Sombor index. From Lemmas 3.10-3.12, we get that the degree sequence of $T$ is $\pi_{1}$ for odd $k$, and $\pi_{2}$ for even $k$. If $3 \leq k \leq \frac{2 n-5}{3}$ and $k$ is odd, then it can be verified that $m_{3,3}=\frac{k-3}{2}, m_{2,3}=\frac{k+3}{2}, m_{1,2}=\frac{k+3}{2}, m_{2,2}=\frac{2 n-3 k-5}{2}$ by Lemma 3.13. Thus we get the desired result (i). By a similar discussion (ii)-(v) can be proved, we omit them here.

In the following, we are going to determine the maximum Sombor index among all the tree of order $n$ with given number of segments.

Theorem 3.15 Let $T$ be a tree in $\mathscr{F}_{n, k}$, we have $S(T) \leq(k-1) \sqrt{1+k^{2}}+\sqrt{4+k^{2}}+$ $\sqrt{5}+2 \sqrt{2}(n-k-2)$ with equality if and only if $T \cong Y_{n, k}$. 
Proof. We can first claim that the tree $T$ which maximizes the Sombor index must be a starlike tree. Otherwise, there is a non-pendant path segment in T. By the path-lifting transform one can get a tree $T^{\prime}$ such that $T^{\prime} \in \mathscr{F}_{n, k}$ and $S\left(T^{\prime}\right)>S(T)$, a contradiction. Now, suppose that $T \nRightarrow Y_{n, k}$, then there are at least two pendant paths $P=v_{1} v_{2} \ldots v_{s} w$ and $P^{\prime}=u_{1} u_{2} \ldots u_{t} w$, where $w$ is the unique branching vertex in $T$, such that $s \geq t \geq 2$. Let $T^{\prime \prime}=T-u_{t-1} u_{t}+u_{t-1} v_{1}$. We have

$$
\begin{aligned}
S\left(T^{\prime \prime}\right)-S(T) & =\sqrt{1^{2}+k^{2}}+2 \sqrt{2}-\sqrt{2^{2}+k^{2}}-\sqrt{5}=2 \sqrt{2}-\sqrt{5}-\frac{3}{\sqrt{1+k^{2}}+\sqrt{4+k^{2}}} \\
& \geq 2 \sqrt{2}-\sqrt{5}-\frac{3}{\sqrt{1+3^{2}}+\sqrt{4+3^{2}}} \approx 0.149086>0
\end{aligned}
$$

a contradiction. Thus we conclude that the maximal tree in $\mathscr{F}_{n, k}$ is isomorphic to the broom $Y_{n, k}$.

It is well-known the fact that the number of vertices of degree two in a tree $T$ is equal to $|V(T)|-k-1$, where $k$ denote the number of segments in $T$. Thus Theorems 3.14 and 3.15 also determine the the minimum and maximum Sombor index of trees of order $n$ with given number of vertices of degree two.

\subsection{The minimum and maximum Sombor indices of trees with given number of branching vertices}

Let $\mathscr{K}_{n, b}$ be the set of trees of orders $n$ with $b$ branching vertices, where $1 \leq b \leq \frac{n-2}{2}$. We determine, in this subsection, the minimum and maximum Sombor indices in $\mathscr{K}_{n, b}$.

Lemma 3.16 If $T$ is a tree which minimizes the Sombor index in $\mathscr{K}_{n, b}$, then $T$ can not contain a branching vertex of degree greater than three.

Proof. Suppose to the contrary that the tree $T$ contains a branching vertex of degree greater than three, and let $u$ be such a vertex whose degree is maximum, i.e, $d_{T}(u)=$ $\Delta(T) \geq 4$. Assume that $P=v_{0} v_{1} \ldots v_{i-1} u\left(=v_{i}\right) v_{i+1} \ldots v_{l+1}$ is a longest path containing $u$ in $T$. Let $d_{T}(u)=x, N_{T}(u)=\left\{v_{i-1}, v_{i+1}, u_{1}, u_{2}, \ldots, u_{x-2}\right\}, d_{T}\left(u_{j}\right)=d_{j}$ and $d_{T}\left(v_{i}\right)=k_{i}$, $i=0,1, \ldots, l+1, j=1,2, \ldots, x-2$. Denote by $w_{1} \in V(T) \backslash V(P)$ a pendant vertex connected to $u$ via $u_{1}$, i.e., there is a path $w_{1} w_{2}, \ldots, w_{r} u$ in $T$, where $r \geq 1$ and $u_{1}$ may coincide with some $w_{i}, i=1,2, \ldots, r$. Let $T^{\prime}=T-u u_{2}+w_{1} u_{2}$.

If $w_{1} \neq u_{1}$, then

$$
S\left(T^{\prime}\right)-S(T)=\sqrt{(x-1)^{2}+k_{i-1}^{2}}+\sqrt{(x-1)^{2}+k_{i+1}^{2}}+\sum_{j=1, j \neq 2}^{x-2} \sqrt{(x-1)^{2}+d_{j}^{2}}
$$




$$
\begin{aligned}
& \quad+\sqrt{2^{2}+d_{T}\left(u_{2}\right)^{2}}+\sqrt{2^{2}+d_{T}\left(w_{2}\right)^{2}}-\sqrt{x^{2}+k_{i-1}^{2}} \\
& -\sqrt{x^{2}+k_{i+1}^{2}}-\sum_{j=1}^{x-2} \sqrt{x^{2}+d_{j}^{2}}-\sqrt{1^{2}+d_{T}\left(w_{2}\right)^{2}} \\
& =\phi\left(2, d_{T}\left(w_{2}\right)\right)-\phi\left(x, k_{i-1}\right)-\phi\left(x, k_{i+1}\right)-\sum_{j=1, j \neq 2}^{x-2} \phi\left(x, d_{j}\right) \\
& +\sqrt{2^{2}+d_{T}\left(u_{2}\right)^{2}}-\sqrt{x^{2}+d_{T}\left(u_{2}\right)^{2}} \quad \text { since } d_{T}\left(u_{2}\right) \leq x \\
& <\phi\left(2, d_{T}\left(w_{2}\right)\right)+\sqrt{2^{2}+d_{T}\left(u_{2}\right)^{2}}-\sqrt{x^{2}+d_{T}\left(u_{2}\right)^{2}} \\
& <\phi(2,2)+\sqrt{2^{2}+d_{T}\left(u_{2}\right)^{2}}-\sqrt{x^{2}+d_{T}\left(u_{2}\right)^{2}} \\
& \leq \phi(2,2)+\sqrt{2^{2}+x^{2}}-\sqrt{x^{2}+x^{2}} \quad \text { by } x \geq 4 \\
& \leq \phi(2,2)+\sqrt{2^{2}+4^{2}}-\sqrt{4^{2}+4^{2}} \quad \\
& =\sqrt{5}-2 \sqrt{2}<0,
\end{aligned}
$$

a contradiction to the choice of $T$.

If $u_{1}=w_{1}$, then

$$
\begin{aligned}
S\left(T^{\prime}\right)-S(T)= & \sqrt{(x-1)^{2}+k_{i-1}^{2}}+\sqrt{(x-1)^{2}+k_{i+1}^{2}}+\sum_{j=3}^{x-2} \sqrt{(x-1)^{2}+d_{j}^{2}} \\
& +\sqrt{2^{2}+d_{T}\left(u_{2}\right)^{2}}+\sqrt{2^{2}+(x-1)^{2}}-\sqrt{x^{2}+k_{i-1}^{2}} \\
& -\sqrt{x^{2}+k_{i+1}^{2}}-\sum_{j=2}^{x-2} \sqrt{x^{2}+d_{j}^{2}}-\sqrt{1^{2}+x^{2}} \\
= & \sqrt{2^{2}+(x-1)^{2}}-\sqrt{1^{2}+x^{2}}-\phi\left(x, k_{i-1}\right)-\phi\left(x, k_{i+1}\right)-\sum_{j=3}^{x-2} \phi\left(x, d_{j}\right) \\
& +\sqrt{2^{2}+d_{T}\left(u_{2}\right)^{2}}-\sqrt{x^{2}+d_{T}\left(u_{2}\right)^{2}} \\
< & \sqrt{2^{2}+x^{2}}-\sqrt{1^{2}+x^{2}}+\sqrt{2^{2}+d_{T}\left(u_{2}\right)^{2}}-\sqrt{x^{2}+d_{T}\left(u_{2}\right)^{2}} \\
\leq & \phi(2, x)+\sqrt{2^{2}+x^{2}}-\sqrt{x^{2}+x^{2}} \\
\leq & \phi(2,4)+\sqrt{2^{2}+4^{2}}-\sqrt{4^{2}+4^{2}} \\
= & 4 \sqrt{5}-\sqrt{17}-4 \sqrt{2}<0,
\end{aligned}
$$

a contradiction to the choice of $T$. Thus, we complete the proof.

Lemma 3.17 If $T$ is a tree which minimizes the Sombor index in $\mathscr{K}_{n, b}$, then the degree sequence of $T$ is $(\underbrace{3,3, \ldots, 3}_{b}, \underbrace{2,2, \ldots, 2}_{n-2 b-2}, \underbrace{1,1, \ldots, 1}_{b+2})$.

Proof. Let $n_{i}$ be the number of vertices of degree $i$ in $T$. In view of Lemma 3.16 we have that there exist only vertices of degree one, two or three in T. By the Handshaking 
Lemma we have $n_{1}+2 n_{2}+3 n_{3}=2|E(T)|=2(|V(T)|-1)=2\left(n_{1}+n_{2}+n_{3}\right)-2$, which leads $n_{1}=n_{3}+2$. Since $n_{3}=b$ we attain the desired result that the degree sequence of $T$ is $(\underbrace{3,3, \ldots, 3}_{b}, \underbrace{2,2, \ldots, 2}_{n-2 b-2}, \underbrace{1,1, \ldots, 1}_{b+2})$.

Let $\mathcal{T}_{n, b}^{1}$ be the set of trees with degree sequence $(\underbrace{3,3, \ldots, 3}_{b}, \underbrace{2,2, \ldots, 2}_{n-2 b-2}, \underbrace{1,1, \ldots, 1}_{b+2})$ and each tree in $\mathcal{T}_{n, b}^{1}$ satisfies that $m_{1,2}=b+2, m_{2,2}=n-3 b-4, m_{2,3}=b+2$ and $m_{3,3}=b-1$ for $b \leq \frac{n-3}{3}$. Let $\mathcal{T}_{n, b}^{2}$ be the set of trees with degree sequence $(\underbrace{3,3, \ldots, 3}_{b}, \underbrace{2,2, \ldots, 2}_{n-2 b-2}, \underbrace{1,1, \ldots, 1}_{b+2})$ and each tree in $\mathcal{T}_{n, b}^{2}$ satisfies that $m_{1,2}=n-2 b-2, m_{1,3}=3 b-n+4, m_{2,3}=n-2 b-2$ and $m_{3,3}=b-1$ for $b>\frac{n-3}{3}$.

Theorem 3.18 Let $T$ be a tree in $\mathscr{K}_{n, b}$.

(i) If $1 \leq b \leq \frac{n-3}{3}$, then $S(T) \geq 3 \sqrt{2}(b-1)+(b+2)(\sqrt{13}+\sqrt{5})+2 \sqrt{2}(n-3 b-4)$ with equality if and only if $T \in \mathcal{T}_{n, b}^{1}$.

(ii) If $\frac{n-3}{3}<b \leq \frac{n-2}{2}$, then $S(T) \geq 3 \sqrt{2}(b-1)+(n-2 b-2)(\sqrt{13}+\sqrt{5})+\sqrt{10}(3 b-n+4)$ with equality if and only if $T \in \mathcal{T}_{n, b}^{2}$.

Proof. The result follows by Lemma 3.13, Lemma 3.16 and Lemma 3.17 and some basic computations.

In the following, we proceed to determine the maximum Sombor index among all the trees of order $n$ with given number of branching vertices.

Lemma 3.19 If $T$ is a tree which maximizes the Sombor index in $\mathscr{K}_{n, b}$, then $T$ contains only pendant vertices and branching vertices.

Proof. Suppose to the contrary that there exists at least one vertex of degree two in $T$. Then there must exist a branching vertex $w$ which adjacent to a vertex $u$ of degree two. Let $N_{T}(u)=\{v, w\}$ and $T^{\prime}=T-u v+v w$. Obviously, $T^{\prime} \in \mathscr{K}_{n, b}$. Let $d_{T}(w)=s+1$, $N_{T}(w)=\left\{u, w_{1}, w_{2}, \ldots, w_{s}\right\}, d_{T}\left(w_{i}\right)=p_{i}$ for $i=1,2, \ldots, s$, and $d_{T}(v)=t$. It holds that

$$
\begin{aligned}
S\left(T^{\prime}\right)-S(T)= & \sum_{i=1}^{s} \sqrt{\left(d_{T}(w)+1\right)^{2}+d_{T}\left(w_{i}\right)^{2}}+\sqrt{1^{2}+\left(d_{T}(w)+1\right)^{2}} \\
& +\sqrt{\left(d_{T}(w)+1\right)^{2}+d_{T}(v)^{2}}-\sum_{i=1}^{s} \sqrt{d_{T}(w)^{2}-d_{T}\left(w_{i}\right)^{2}} \\
& -\sqrt{2^{2}+d_{T}(w)^{2}}-\sqrt{2^{2}+d_{T}(v)^{2}} \\
= & \sum_{i=1}^{s} \sqrt{(s+1)^{2}+p_{i}^{2}}+\sqrt{1^{2}+(s+1)^{2}}+\sqrt{(s+1)^{2}+t^{2}}
\end{aligned}
$$




$$
\begin{aligned}
& -\sum_{i=1}^{s} \sqrt{s^{2}+p_{i}^{2}}-\sqrt{2^{2}+s^{2}}-\sqrt{2^{2}+t^{2}} \\
> & \sqrt{1^{2}+(s+1)^{2}}-\sqrt{2^{2}+s^{2}}+\sqrt{(s+1)^{2}+t^{2}}-\sqrt{2^{2}+t^{2}}>0,
\end{aligned}
$$

a contradiction. Thus the proof is completed.

Lemma 3.20 If $T$ is a tree which maximizes the Sombor index, then $T$ contains at most one vertex greater than three.

Proof. Suppose to the contrary that there are two vertices $u$ and $v$ with $d_{T}(v)=t \geq$ $d_{T}(u)=l \geq 4$, and let $N(u)=\left\{u_{1}, u_{2}, \ldots, u_{l}\right\}, N(v)=\left\{v_{1}, v_{2}, \ldots, v_{t}\right\}, d\left(u_{i}\right)=x_{i}, d\left(v_{i}\right)=$ $y_{i}$, and $u_{1}$ locate in the path connecting $u$ to $v\left(u_{1}\right.$ may coincide with $\left.v\right)$. Then $d_{T}\left(u_{1}\right) \geq 2$ and $d_{T}\left(u_{i}\right)=x_{i} \geq 1$ for $i=2,3, \ldots, l$. Let $\varphi(x, y)=\sqrt{(x+l-3)^{2}+y^{2}}-\sqrt{x^{2}+y^{2}}$. Then by calculating the derivative of the function one can see that $\varphi(x, y)$ is strictly decreasing with $y$ for fixed $x$. From the inequality of arithmetic and geometric means $\sqrt{x y} \leq \frac{x+y}{2} \leq \sqrt{\frac{x^{2}+y^{2}}{2}}$ one can get that $\sqrt{(t+l-3)^{2}+t^{2}} \geq \frac{2 t+l-3}{\sqrt{2}}$, which will be useful in the following proof. We can construct a new tree $T^{\prime}=T-\left\{u u_{i}: 4 \leq i \leq l\right\}+\left\{v u_{i}\right.$ : $4 \leq i \leq l\}$ such that $T^{\prime} \in \mathscr{K}_{n, b}$.

Case 1. $u v \notin E(T)$.

Let $\frac{\sqrt{2}}{2}(l+1)(l-3)-2 \sqrt{l^{2}+1}-\sqrt{l^{2}+4}+2 \sqrt{10}+\sqrt{13}$, then for $x \geq 4$ we have

$$
h_{1}^{\prime}(x)=\sqrt{2}(x-1)-\frac{2 x}{2 \sqrt{x^{2}+1}}-\frac{x}{2 \sqrt{x^{2}+4}}>\sqrt{2}(x-1)-\frac{3 x}{2 \sqrt{x^{2}+1}}>3 \sqrt{2}-\frac{3}{2}>0,
$$

which means $h_{1}(x)$ is strictly increasing with $x \geq 4$. Then we have

$$
\begin{aligned}
S\left(T^{\prime}\right)-S(T) & =\sum_{i=1}^{3} \sqrt{3^{2}+x_{i}^{2}}+\sum_{i=4}^{l} \sqrt{(t+l-3)^{2}+x_{i}^{2}}+\sum_{i=1}^{t} \sqrt{(t+l-3)^{2}+y_{i}^{2}} \\
& -\sum_{i=1}^{l} \sqrt{l^{2}+x_{i}^{2}}-\sum_{i=1}^{t} \sqrt{t^{2}+y_{i}^{2}}=\sum_{i=4}^{l} \varphi\left(t, x_{i}\right)+\sum_{i=1}^{t} \varphi\left(t, y_{i}\right)-\sum_{i=1}^{3} \varphi\left(3, x_{i}\right) \\
& >\sum_{i=4}^{l} \varphi(t, t)+\sum_{i=1}^{t} \varphi(t, t)-\varphi(3,2)-2 \varphi(3,1) \\
& =(t+l-3) \sqrt{(t+l-3)^{2}+t^{2}}-(l-3) \sqrt{l^{2}+t^{2}}-\sqrt{2} t^{2}-\varphi(3,2) \\
& -2 \varphi(3,1) \geq \frac{(t+l-3)(2 t+l-3)}{\sqrt{2}}-(l-3) \sqrt{l^{2}+t^{2}}-\sqrt{2 t^{2}}-\varphi(3,2)-2 \varphi(3,1) \\
& =(l-3)\left(\sqrt{2 t^{2}}-\sqrt{l^{2}+t^{2}}\right)+\frac{\sqrt{2}(t+l-3)(l-3)}{2}-\varphi(3,2)-2 \varphi(3,1) \\
\geq & \frac{\sqrt{2}(t+l-3)(l-3)}{2}-\varphi(3,2)-2 \varphi(3,1) \geq \frac{\sqrt{2}(4+l-3)(l-3)}{2}-\varphi(3,2)-2 \varphi(3,1) \\
& =\frac{\sqrt{2}}{2}(l+1)(l-3)-2 \sqrt{l^{2}+1}-\sqrt{l^{2}+4}+2 \sqrt{10}+\sqrt{13}=h_{1}(l)
\end{aligned}
$$




$$
\geq h_{1}(4)=\frac{5 \sqrt{2}}{2}-2 \sqrt{5}+2 \sqrt{10}+\sqrt{13}-2 \sqrt{17} \approx 0.747293>0,
$$

a contradiction.

Case 2. $u v \in E(T)$.

Without loss of generality we set $u_{1}=v$ and $v_{1}=u$. Let $h_{2}(x)=\frac{\sqrt{2}}{2} x(x-3)-$ $2 \sqrt{x^{2}+1}+2 \sqrt{10}$. It is easy to verify that $h_{2}(x)$ is strictly increasing with $x \geq 4$. Then

$$
\begin{aligned}
S\left(T^{\prime}\right)-S(T)= & \sum_{i=2}^{3} \sqrt{3^{2}+x_{i}^{2}}+\sqrt{3^{2}+(t+l-3)^{2}}+\sum_{i=4}^{l} \sqrt{(t+l-3)^{2}+x_{i}^{2}} \\
& +\sum_{i=2}^{t} \sqrt{(t+l-3)^{2}+y_{i}^{2}}-\sum_{i=2}^{l} \sqrt{l^{2}+x_{i}^{2}}-\sum_{i=2}^{t} \sqrt{t^{2}+y_{i}^{2}}-\sqrt{t^{2}+l^{2}} \\
> & \sum_{i=4}^{l} \varphi\left(t, x_{i}\right)+\sum_{i=2}^{t} \varphi\left(t, y_{i}\right)-\sum_{i=2}^{3} \varphi\left(3, x_{i}\right) \\
> & \sum_{i=4}^{l} \varphi(t, t)+\sum_{i=2}^{t} \varphi(t, t)-2 \varphi(3,1) \\
= & (t+l-4) \sqrt{(t+l-3)^{2}+t^{2}}-(l-3) \sqrt{l^{2}+t^{2}}-\sqrt{2} t(t-1)-2 \varphi(3,1) \\
\geq & \frac{(t+l-4)(2 t+l-3)}{\sqrt{2}}-(l-3) \sqrt{l^{2}+t^{2}}-\sqrt{2} t(t-1)-2 \varphi(3,1) \\
= & (l-3)\left(\sqrt{2} t-\sqrt{l^{2}+t^{2}}\right)+\frac{\sqrt{2}(t+l-4)(l-3)}{2}-2 \varphi(3,1) \\
\geq & \frac{\sqrt{2}(t+l-4)(l-3)}{2}-2 \varphi(3,1) \geq \frac{\sqrt{2}(4+l-4)(l-3)}{2}-2 \varphi(3,1) \\
= & \frac{\sqrt{2}}{2} l(l-3)-2 \sqrt{l^{2}+1}+2 \sqrt{10}=h_{2}(l) \\
\geq & h_{2}(4)=2 \sqrt{2}-2 \sqrt{17}+2 \sqrt{10} \approx 0.906771>0,
\end{aligned}
$$

a contradiction to the maximality of $T$ in $\mathscr{K}_{n, b}$.

Therefore, we complete the proof.

Lemma 3.21 If $T$ is a tree in $\mathscr{K}_{n, b}$ with the maximum Sombor index, then $m_{3, n-2 b+1}(T)=$ 1 for $1 \leq b \leq \frac{n-3}{2}$.

Proof. Form Lemma 3.19 and Lemma 3.20 we have that the degree sequence of $T$ is $(n-2 b+1, \underbrace{3,3, \ldots, 3}_{b-1}, \underbrace{1,1, \ldots, 1}_{n-b})$. Let $u$ be the maximum degree vertex of $T$, i.e. $\Delta(T)=$ $d_{T}(u)=n-2 b+1 \geq 4$. Suppose to the contrary that $m_{3, n-2 b+1} \geq 2$, then there are at least two branching vertices adjacent to $u$. As a matter of fact, one can always choose two branching vertices $w$ and $v$ which satisfy that $d_{T}(w)=d_{T}(v)=3$, where $w$ has a pendant vertices $w_{1}$ as neighbor and $u v \in E(T)$. Let $T^{\prime}=T-u v-w w_{1}+u w_{1}+w u$. It is easy to 
see that the degree sequences of $T$ and $T^{\prime}$ are the same, implying that the obtained tree $T^{\prime}$ also belongs to $\mathscr{K}_{n, b}$. We have

$$
\begin{aligned}
S\left(T^{\prime}\right)-S(T) & =\sqrt{(n-2 b+1)^{2}+1^{2}}+\sqrt{3^{2}+3^{2}}-\sqrt{(n-2 b+1)^{2}+3^{2}}-\sqrt{3^{2}+1^{2}} \\
& \geq \sqrt{4^{2}+1^{2}}+\sqrt{3^{2}+3^{2}}-\sqrt{4^{2}+3^{2}}-\sqrt{3^{2}+1^{2}} \\
& =\sqrt{17}+3 \sqrt{2}-5-\sqrt{10} \approx 0.2035>0,
\end{aligned}
$$

a contradiction to our choice of $T$. Thus, we complete the proof.

Let $\mathcal{T}_{n, b}^{3}$ be the set of trees in $\mathscr{K}_{n, b}$ with degree sequence $(n-2 b+1, \underbrace{3,3, \ldots, 3}_{b-1}, \underbrace{1,1, \ldots, 1}_{n-b})$ and satisfying that $m_{1,3}=b, m_{3,3}=b-2, m_{1, n-2 b+1}=n-2 b$ and $m_{3, n-2 b+1}=1$.

Theorem 3.22 Let $T$ be a tree of order $n$ and having $b$ branching vertices, where $1 \leq b \leq$ $\frac{n-2}{2}$, then $S(T) \leq 3(b-2) \sqrt{2}+b \sqrt{10}+(n-2 b) \sqrt{1+(n-2 b+1)^{2}}+\sqrt{9+(n-2 b+1)^{2}}$, the equation holds if and only if $T \in \mathcal{T}_{n, b}^{3}$.

Proof. Let $T$ be a tree in $\mathscr{K}_{n, b}$ with the maximum Sombor index. According to Lemma 3.19 and Lemma 3.20 we have that the degree sequence of $T$ is $(n-2 b+1, \underbrace{3,3, \ldots, 3}_{b-1}, \underbrace{1,1, \ldots, 1}_{n-b})$. For $b=\frac{n-2}{2}$, the result follows immediately. Furthermore, for $1 \leq b \leq \frac{n-3}{2}$, by Lemma 3.21 we can claim that $T \in \mathcal{T}_{n, b}^{3}$. It is easy to get that the Sombor index of trees in $\mathcal{T}_{n, b}^{3}$ is $3(b-2) \sqrt{2}+b \sqrt{10}+(n-2 b) \sqrt{1+(n-2 b+1)^{2}}+\sqrt{9+(n-2 b+1)^{2}}$. Therefore, we complete the proof.

Acknowledgment: This work is partially supported by the Hunan Provincial Natural Science Foundation of China (2020JJ5612) and the Hunan Education Department Foundation (18A382,19B064).

\section{References}

[1] A. Ali, I. Gutman, E. Milovanović, I. Milovanović, Sum of powers of the degrees of graphs: Extremal results and bounds, MATCH Commun. Math. Comput. Chem. 80 (2018) 5-84.

[2] A. Ali, L. Zhong, I. Gutman, Harmonic index and its generalizations: Extremal results and bounds, MATCH Commun. Math. Comput. Chem. 81 (2019) 249-311.

[3] B. Borovićanin, K. C. Das, B. Furtula, I. Gutman, Bounds for Zagreb indices, MATCH Commun. Math. Comput. Chem. 78 (2017) 17-100. 
[4] B. Borovićanin, On the extremal Zagreb indices of trees with given number of segments or given number of branching vertices, MATCH Commun. Math. Comput. Chem. 74 (2015) 57-79.

[5] R. A. Brualdi, J. L. Goldwasser, Permanent of the Laplacian matrix of trees and bipartite graphs, Discr. Math. 48 (1984) 1-21.

[6] S. Bermudo, J. Nápoles, J. Rada, Extremal trees for the Randić index with given domination number, Appl. Math. Comput. 375 (2020) \#125122.

[7] R. Cruz, J. Rada, Extremal values of the Sombor index in unicyclic and bicyclic graphs, J. Math. Chem. 59 (2021) 1098-1116.

[8] R. Cruz, J. Rada, The path and the star as extremal values of vertex-degree-based topological indices among trees, MATCH Commun. Math. Comput. Chem. 82 (2019) $715-732$.

[9] R. Cruz, I. Gutman, J. Rada, Sombor index of chemical graphs, Appl. Math. Comput. 399 (2021) \#126018.

[10] H. Deng, Z. Tang, R. Wu, Molecular trees with extremal values of Sombor indices, Int. J. Quantum Chem. 121 (2021) \# 26622.

[11] I. Gutman, Geometric approach to degree-based topological indices: Sombor indices, MATCH Commun. Math. Comput. Chem. 86 (2021) 11-16.

[12] M. Goubko, I. Gutman, Degree-based topological indices: Optimal trees with given number of pendents, Appl. Math. Comput. 240 (2014) 387-398.

[13] Q. Fan, S. Li, Q. Zhao, Extremal values on the harmonic number of trees, Int. J. Comput. Math. 92 (2015) 2036-2035.

[14] Y. Hou, J. Li, Bounds on the largest eigenvalues of trees with a gvien size of matching, Lin. Algebra Appl. 342 (2002) 203-217.

[15] H. Lin, On segments, vertices of degree two and the first Zagreb index of trees, MATCH Commun. Math. Comput. Chem. 72 (2014) 825-834.

[16] T. Vetrík, S. Balachandran, General multiplicative Zagreb indices of trees, Discr. Appl. Math. 247 (2018) 341-351.

[17] Z. Wang, Y. Mao, Y. Li, B. Furtula, On relations between Sombor and other degree based indices, J. Appl. Math. Comput., in press. doi:10.1007/s12190-021-01516-x.

[18] Y. Yao, M. Liu, X. Gu, Unified extremal results for vertex-degree-based graph invariants with given diameter, MATCH Commun. Math. Comput. Chem. 82 (2019) 699-714. 